\title{
Experimental and Numerical investigation of a cross flow air-to-water heat pipe-based heat exchanger used in waste heat recovery
}

\author{
Joao Ramos ${ }^{\mathrm{a}}$, Alex Chong ${ }^{\mathrm{a}}$, Hussam Jouhara ${ }^{\mathrm{b}}$ \\ ${ }^{a}$ Faculty of Computing, Engineering and Science, University of South Wales, Pontypridd, CF37 1DL, \\ United Kingdom \\ ${ }^{\mathrm{b}}$ Institute of Energy Futures, RCUK Centre for Sustainable Energy Use in Food Chains, Brunel \\ University London, Uxbridge, UB8 3PH, United Kingdom
}

* Corresponding author. Email: hussam.jouhara@brunel.ac.uk; Tel. +44(0) 1895267806

\begin{abstract}
This paper applies CFD modelling and numerical calculations to predict the thermal performance of a cross flow heat pipe based heat exchanger. The heat exchanger under study transfers heat from air to water and it is equipped with six water-charged wickless heat pipes, with a single-pass flow pattern on the air side (evaporator) and two flow passes on the water side (condenser). For the purpose of CFD modelling, the heat pipes were considered as solid devices of a known thermal conductivity which was estimated by experiments conducted on the exact same heat pipe configuration under an entire testing range. The CFD results were compared with the experimental and the numerical results and it was found that the modelling predictions are within $10 \%$ of the experimental results.
\end{abstract}

Keywords heat pipe; heat exchanger; CFD modelling; heat recovery.

\section{Nomenclature}

Abbreviations

$H P H X \quad$ Heat pipe-equipped heat exchanger

HVAC Heating, Ventilation and Air Conditioning

NTU Number of Transfer Units

TSHX Thermosyphon-equipped heat exchanger

VOF Volume of Fraction (CFD method)

$\mathrm{Pr} \quad$ Prandtl number

$\mathrm{Nu} \quad$ Nusselt number

Re Reynolds number

Symbols

A Area

C Heat Capacity Rate $\left(\dot{m} \times c_{p}\right)$

$c_{p} \quad$ Specific heat at constant pressure

$C_{r} \quad$ Heat Capacity Ratio $\left(C_{e} / C_{v}\right)$

$c_{s f} \quad$ Constant/coefficient dependent on surface-liquid combination

$d \quad$ Characteristic dimension

$g \quad$ Acceleration of gravity

$h \quad$ Heat transfer coefficient

$h_{f g} \quad$ Latent heat

$k \quad$ Thermal Conductivity

$Q \quad$ Heat Transfer Rate

$q^{\prime \prime} \quad$ Heat flux

$r \quad$ Radius

T Temperature

$U \quad$ Overall Heat Transfer Coefficient

$\Delta \quad$ Difference

$\Delta T_{L M} \quad$ LMTD - Logarithmic Mean Temperature Difference

$\varepsilon \quad$ Effectiveness

$\mu \quad$ Static viscosity of the liquid phase 


$\begin{array}{ll}\pi & \text { Pi } \\ \rho & \text { Density } \\ \sigma & \text { Surface tension }\end{array}$

$\begin{array}{ll}\text { Subscripts } & \\ a c t & \text { Actual } \\ b & \text { Boiling } \\ c & \text { Condenser } \\ c d / \text { cond } & \text { Condensation } \\ e & \text { Evaporator } \\ h & \text { Heat transfer coefficient } \\ i & \text { Inside } \\ i n & \text { inner } \\ k & \text { Conduction } \\ l & \text { Liquid phase } \\ m i n & \text { Minimum } \\ n & \text { Number of pipes } \\ o & \text { Outside } \\ s & \text { Surface } \\ s a t & \text { Saturation } \\ t & \text { Total } \\ t s & \text { thermosyphon }\end{array}$

\section{$1 \quad$ Introduction}

Heat exchangers are commonly employed as heat recovery devices to reuse the wasted heat energy from exhaust outlets so it may be furtherly reused or stored for a later use. According to the research of Haddad et al. [1] 90\% of the wasted heat energy is found at low to medium-grade heat applications (temperatures from 100 to $400^{\circ} \mathrm{C}$ ), as can be seen in Figure 1. It is in this environment that heat pipeequipped heat exchangers are finding wide use due to an array of advantages ranging from a complete flow separation, great redundancy and ease of maintenance. All of the advantages are a direct result of the mechanism of phase change happening within the heat pipe [2].

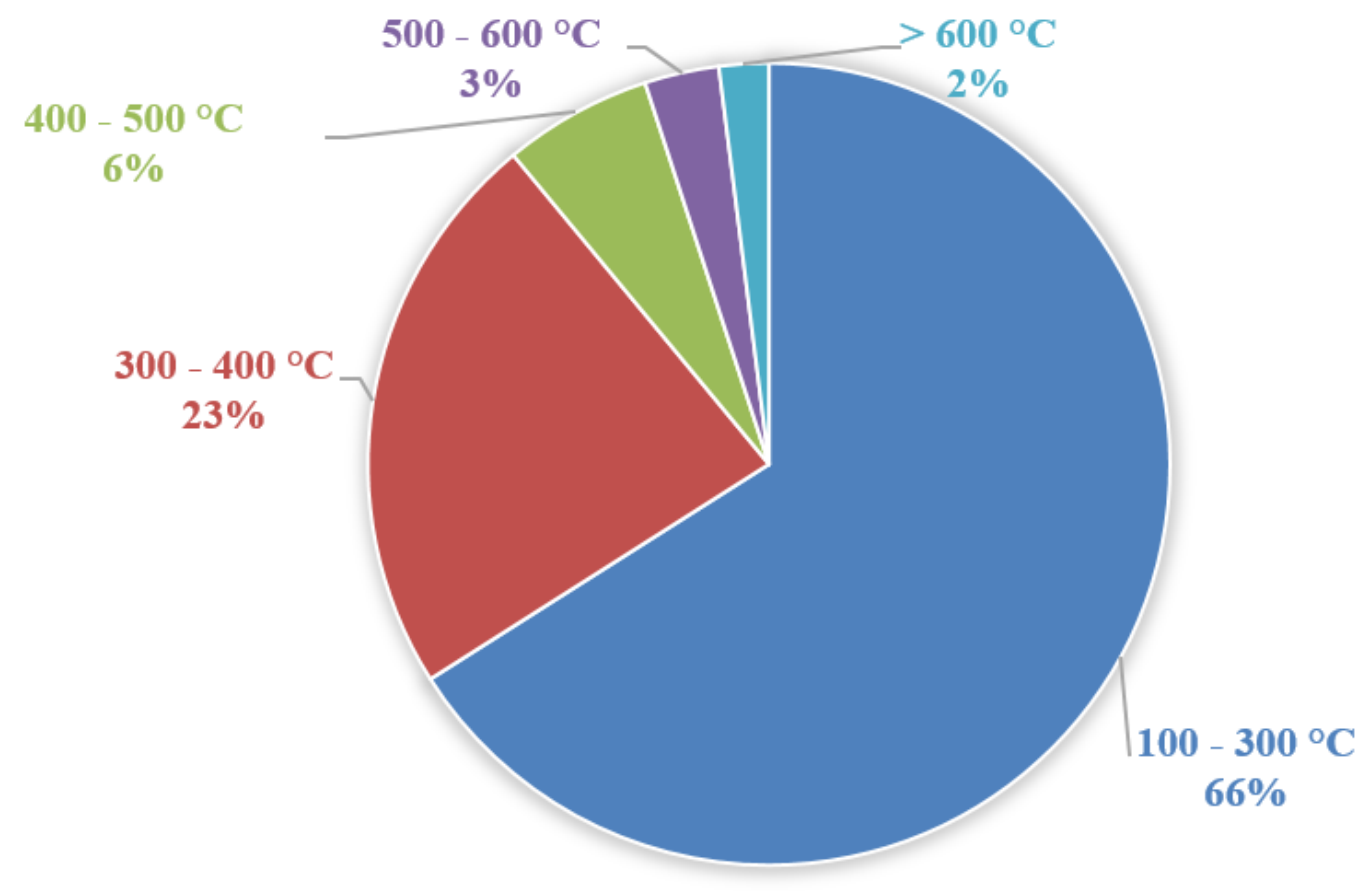


Figure 1: Waste heat energy by temperature range (adapted from [1])

\subsection{Literature Review}

Heat pipes were initially developed by NASA as effective heat sinks to cool down small-scale electronic equipment in space [3], while nowadays they are commonly used for cooling purposes of electronic equipment from mobile phones to CPUs $[4,5,6]$. A heat pipe consists of a hermeticallysealed tube filled with a small mass of saturated working fluid that exists in liquid and vapour form and occupies the whole of the internal volume of the tube. Applying heat to one end of the heat pipe will cause the working fluid inside the pipe to boil and, due to the lower density, to travel in vapour form towards the cooler end of the pipe, where it condenses and gives away the absorbed latent heat that was collected in the evaporator section; thus completing the thermal cycle [7]. A representation of the heat pipe working cycle can be seen in Figure 2. Due to the high effective thermal conductivity of these devices at essentially constant temperature throughout its length $[8,9]$, heat pipes have been referred as superconductors. Their effective conductivity can easily be several orders of magnitude greater than pure conduction through a solid metal $[5,10,11]$

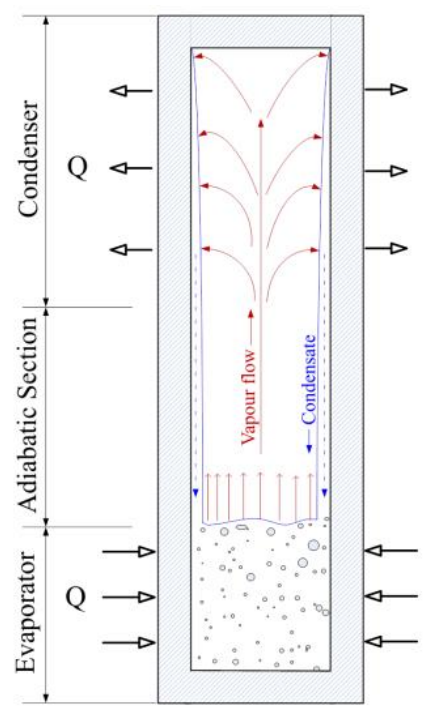

Figure 2: Schematic of a working heat pipe

The small heat pipes used in electronic applications are equipped with a porous wick structure, which allows them to function in any orientation, provided there is a difference in temperature between both sides of the pipe [7, 9]. However, the heat pipe does not require a wick in order to function properly; as long as the evaporator section is located below the condenser section, the condensate working fluid is pushed back to the evaporator through the force of gravity. For that reason, wickless heat pipes are also known as gravity-assisted heat pipes or two-phase closed thermosyphons [9]. The term "thermosyphon" is used throughout the paper and refer to the devices employed in this study.

Thermosyphon-equipped heat exchangers (TSHE) offer many advantages when used as waste heat recovery devices, such as an increased redundancy and reliability, ease of cleaning, no additional power input to the system, reduced risk of cross-contamination and no moving parts; all advantages widely highlighted in the literature surveyed $[12,13,14,15,16,17]$. There is much literature available in heat exchangers equipped with heat pipes (HPHX) used in Heating, Ventilation and Airconditioning applications (HVAC) [5, 10, 18, 19, 20], as well as heat recovery [21, 22, 23]. For low to medium grade heat, consisting of temperatures above $150^{\circ} \mathrm{C}$, Noie [24] presents an analytical method of characterising the HPHX using the Effectiveness ( $\varepsilon$-NTU method) to predict the performance of the heat exchanger. The same approach is taken by Danielewicz et al. [25], Jouhara \& Merchant [26] and Han \& Zou [8] with the aid of a computer coding that predicts the effectiveness depending on different inlet conditions. All the surveyed papers refer to air-to-air HPHX even if it is mentioned by Noie that the $\varepsilon$-NTU method theory applies even if different fluids are used on the shell side. 
The development of CFD codes for simulation of heat pipes and heat pipe heat exchangers is a relatively new field of research. It has been receiving renewed interest due to recent advances in computing which allow the simulation of the phase change within the thermosyphon. However, a divide seems to exist in the reviewed literature; the authors either focus solely into the CFD simulation of the phase change process in the thermosyphon or on the behaviour of the fluid on the shell side of a HPHX.

The simulation of heat pipes through CFD is a fairly novel field of research, made possible due to the increase in computational power of modern computers. Alizadehdakhel et al. [27] and Fadhl et al. [28] have both successfully modelled the thermosyphon using a custom volume of fraction (VOF) code in Fluent, a popular CFD release. Both works depicted a 2 dimensional study and proved that the software is capable of simulating the phase change process within a single heat pipe during the evaporation and condensation processes albeit after a long processing time. More recently, the same method has been successfully applied to a 3D model to simulate Geyser boiling in the heat pipe [29]. Geyser boiling usually takes place at low heat input, when a large amount of evaporated fluid bubbles starts to form below the liquid bulk. When the pressure difference between the bubble and the liquid bulk becomes too great, the liquid is projected into the top of the thermosyphon [30, 31].

CFD has also been used to calculate the optimum filling ratio for a thermosyphon by calculating the quantity that will allow the shortest response time and lowest thermal resistance [32]. According to Shabgard et al. [32], it is recommended that an extra 5-10\% of fluid is inserted in the pipe to prevent breakdown of the liquid film from the thermosyphon wall. A three-dimensional numerical study simulating multi-phase flow inside horizontally oriented heat pipes was conducted by Hughes et al. [33] for steady-state conditions. In this study, a multiphase flow with coupled heat and mass transfer was used. In order to predict the performance of the heat pipes, the effectiveness of the heat exchanger was determined through an experimental study. A good correlation was found between the results from the CFD model and the experimental results for the same operating conditions.

As mentioned previously, a progression is being observed in the application of CFD to the simulation of thermosyphons, however there is not much literature on the study of the entire HPHX or TSHX. Selma et al. [34] have designed a working model of a heat exchanger equipped with heat pipes using OpenFOAM, an open-access CFD release, in order to improve the energy efficiency of an existing model. A 3-dimensional simulation of the external flow surrounding the pipes was created and used the outer wall of the pipes as a constant temperature boundary condition gathered from industrial practice. The results proved very satisfactory and correlated very well with both experimental data and a commercial CFD release. Peng et al [35] conducted a CFD study on the effect of fin shape on the air-side heat transfer performance of a fin-plate thermosyphon used in electronics cooling. The simulation focused solely on the air side and results from the CFD model were within $15 \%$ error of the experimental results. CFD has also been employed to simulate the feasibility of installing heat pipes within a wind tower. In a study by Calautita et al [36], the heat pipes were modelled as having a constant surface temperature, a reasonable assumption taking into account there is little difference in the temperature of the working fluid inside the pipe. The results showed that the incorporation of heat pipes in this application is capable of improving the reduction in inlet air temperature.

It appears that there is a gap in the form of o attempt made at simulating the thermosyphons and the heat exchanger in the same simulation. Other than the VOF method, there were no other recommendations in terms of alternative methods of simulating the thermosyphons using, for example, the thermal network analogy. The author therefore recommends the application of the thermal network analogy in order to predict the thermal conductivity of the thermosyphons and feeding that value as a boundary condition into the CFD model of the TSHX. Mroué et al. [37] conducted a study similar to the one present in this work, in which the flow on the hot side of the TSHX was allowed to return in order to make contact with the tubes where film boiling takes place. The thermal network analysis is also used in conjunction with the $\varepsilon$-NTU method in order to accurately predict the behaviour of the TSHX. 
The objective of this paper is to implement the analytical and theoretical background analysis of thermosyphons with CFD simulations, by assuming that the heat pipes are solid devices of a constant conductivity. The conductivity is predicted using adapted versions of equations found in literature. The results shall prove that the thermal resistance analogy within the heat pipe can be extended to 3dimensional CFD simulations.

At first the theoretical modelling of thermosyphons is presented in detail. It is followed by the experimental set up and the designing conditions of the CFD model. The results are then presented and compared.

\section{Theoretical Analysis}

In terms of predicting the performance of a heat exchanger equipped with thermosyphons, there are quite a few examples in the literature; Azad \& Geoola [38] and Kays \& London [39] were some of the first to report the use of the effectiveness-Number of Transfer Units ( $\varepsilon$-NTU) method to predict the performance of a heat exchanger equipped with thermosyphons to great effect. Even to this day authors continue using the same approach as it has provided satisfactory results; Lukitobudi et al. [40] used it in an approach to recovering waste heat in bakeries, Noie [24] used it in an investigation of an air-to-air heat exchanger used in heat recovery, and Jouhara \& Merchant [26] reported the same in their multi-use apparatus.

\subsection{Predicting the performance of a single thermosyphon}

A thermosyphon is, in many ways, a miniature heat exchanger; so it is only natural to approach it the same way a heat exchanger is approached. The most reported method of predicting the performance of a thermosyphon is through the thermal network analogy [7, 9, 13, 37], also approached in this study. In this analogy, the thermosyphon is broken down into its inner thermal resistances, conduction, boiling, condensation, etc.

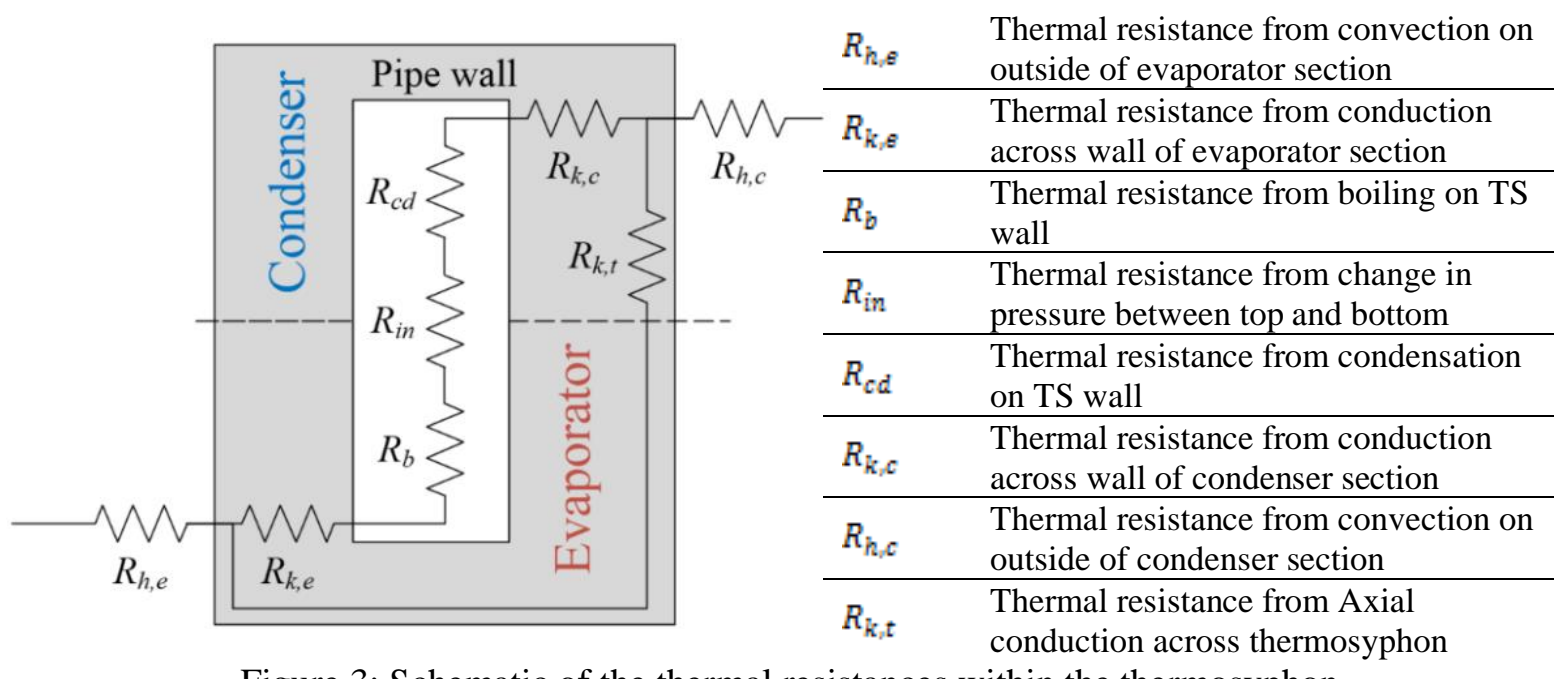

Figure 3: Schematic of the thermal resistances within the thermosyphon

Treating the circuit displayed in Figure 3 as an electrical circuit; and neglecting the axial thermal conductivity, the total thermal resistance for the thermosyphon is found through equation (1).

$$
R_{T}=R_{h_{i} e}+R_{k_{i e}}+R_{b}+R_{\text {in }}+R_{\text {ced }}+R_{k_{i j}}+R_{h_{i} c}
$$

The axial thermal conductivity along thin-walled thermosyphons with long adiabatic sections may be considered negligible $[7,13]$ therefore simplifying the expression to a simple addition. In the case of a heat pipe, which is equipped with a wick structure, an additional parallel network of thermal resistances is added which includes the convection to enter the wick and the axial conductivity along the length of the device. 


\subsection{Conduction through the thermosyphon walls}

The flow of heat through the thermosyphon starts with heat entering the heat pipe through the evaporator wall. The thermal resistance at the evaporator wall is deducted from conduction through a hollow pipe wall:

$$
\dot{Q}_{k}=\frac{2 \pi k l \Delta T}{\ln \left(r_{\text {out }}-r_{\text {in }}\right)} \quad \therefore \quad R_{k}=\frac{\ln \left(r_{\text {out }}-r_{\text {in }}\right)}{2 \pi k l}
$$

Where $k$ is the thermal conductivity of the encasing material $(\mathrm{W} / \mathrm{mK}), l$ is the length of the pipe in contact with the hot air flow (m), $r_{\text {out }}-r_{\text {in }}$ represent the tube thickness (m) and $\Delta T$ is the difference in temperature between the inside and the outside of the pipe $\left({ }^{\circ} \mathrm{C}\right)$. This applies to the evaporator and to the condenser section equally but with different values for the inner and outer temperatures. It is mainly affected by the area of exposure and the conductivity of the material.

\subsection{Convection outside the thermosyphon}

Looking at either section, heat transfer through convection from the surrounding medium to the pipe is given by the following equations for the evaporator section and the condenser section, respectfully:

$$
\hat{Q}_{h}=h A \Delta T \quad \therefore \quad R_{h_{i} e}=\frac{1}{h_{e} A_{e}} \quad \text { and } \quad R_{h_{e} c}=\frac{1}{h_{c} A_{c}}
$$

$h$ refers to the heat transfer coefficient $\left(\mathrm{W} / \mathrm{m}^{2} \mathrm{~K}\right)$ between the fluid and the solid surface, which in this case is air-carbon steel for $h_{\theta}$ and water-carbon steel for $h_{e} . A$ is the exposed surface area $\left(\mathrm{m}^{2}\right)$ and $\Delta T$ the difference in temperature between the flow and the surface area.

The heat transfer coefficient is derived from the Nusselt number through the expression:

$$
N u=\frac{h d}{k}
$$

Where $\mathrm{h}$ represents the heat transfer coefficient $(\mathrm{W} / \mathrm{m} 2 \mathrm{~K}), \mathrm{d}$ the characteristic dimension $(\mathrm{m})$ and $\mathrm{k}$ the thermal conductivity $(\mathrm{W} / \mathrm{mK})$ of the surrounding fluid. The Nusselt number is a function of the flow conditions, in particular the turbulence and will therefore be different in the evaporator section and in the condenser section.

\subsubsection{Convection outside the thermosyphon's condenser section}

A cut-section of the condenser section of the heat exchanger under study is schematically represented in Figure 4.

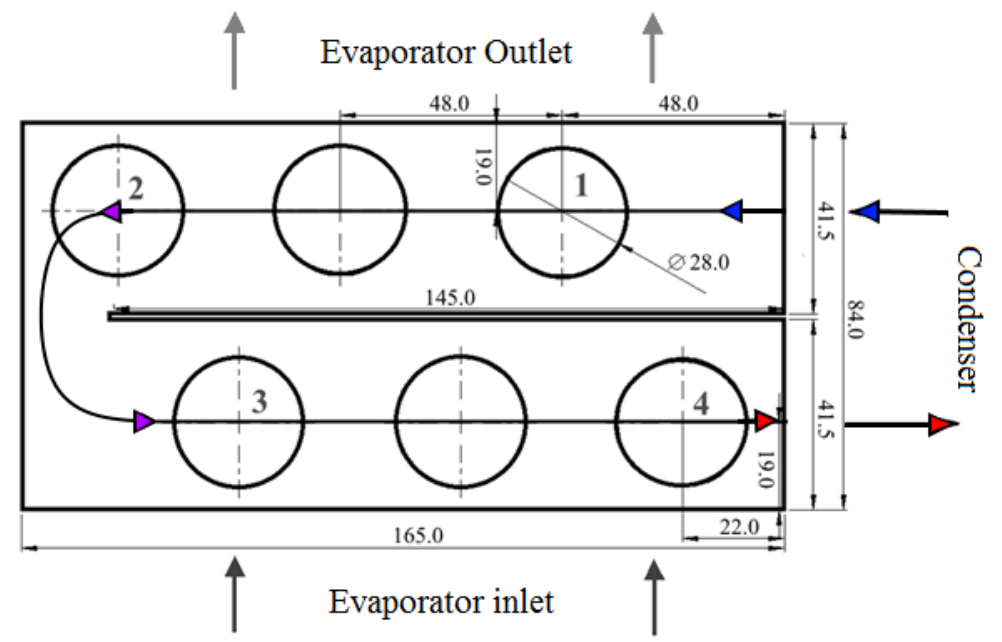

Figure 4: Cross-section of the condenser section of the heat exchanger under study (all dimensions in $\mathrm{mm}$ ) 
Water flows through each thermosyphon one by one following a u-shaped path. The heat transfer by convection over a vertical cylinder in cross-flow has been extensively studied in literature and it applies to the case at hand. The correlation used for external flow over a single cylinder is that of Zhukauskas [41]:

$$
\begin{gathered}
N u=C \operatorname{Re}^{m} \operatorname{Pr}^{n}\left(\frac{P r}{P r_{S}}\right)^{1 / 4} \\
{[0.7<\operatorname{Pr}<500]} \\
{\left[1<\operatorname{Re}<1 \times 10^{6}\right]}
\end{gathered}
$$

The constants $C$ and $m$ are functions of the turbulence in the vicinity of the cylinder and are available in Table 1. All fluid properties are evaluated at the arithmetic mean of the fluid inlet and outlet temperatures except for the properties marked with an $s$, which are evaluated at the boundary between the solid and the fluid.

Table 1: Constants of equation (5) for a circular cylinder in cross flow [41] - excerpt

\begin{tabular}{ccc}
$R e$ & $C$ & $m$ \\
\hline $1-40$ & 0.75 & 0.4 \\
$40-1000$ & 0.51 & 0.5 \\
$10^{3}-2 \times 10^{5}$ & 0.26 & 0.6
\end{tabular}

\subsubsection{Convection outside the thermosyphon's evaporator section}

In the evaporator section the thermosyphons are organised into two lines of three thermosyphons each as seen in Figure 4. Equation (5) is not applicable to the evaporator section due to the higher volume of pipes, and Zhukauskas' [41] correlation for a range of vertical tubes in a staggered arrangement is preferred. The expression has the form:

$$
\begin{gathered}
N u=C_{1} C_{2} \operatorname{Re}_{\max }^{m} \operatorname{Pr}^{0.36}\left(\frac{P r}{P r_{s}}\right)^{1 / 4} \\
{\left[N_{L} \geq 20\right]} \\
{\left[2000<R e_{\max }<40,000\right]} \\
{[P r \geq 0.7]}
\end{gathered}
$$

All properties of the fluids used in equation (6) are evaluated at the mean film temperature except the properties marked with an $s$, which are evaluated at the boundary temperature. $C_{1}$ and $m$ depend on the geometry of the tube bundle and are taken from Table 2, $C_{2}$ is the correction factor used in case

\begin{tabular}{|c|c|c|c|}
\hline Configuration & $R e_{\max }$ & $C_{1}$ & $m$ \\
\hline Staggered & $10-10^{2}$ & 0.90 & 0.40 \\
\hline Staggered & $10^{2}-10^{3}$ & \multicolumn{2}{|c|}{$\begin{array}{l}\text { Approximate as a single } \\
\text { (isolated) cylinder }\end{array}$} \\
\hline Staggered $S_{T} / S_{L}<2$ & $10^{3}-2 \times 10^{5}$ & $0.35\left(S_{T} / S_{L}\right)^{1 / 5}$ & 0.60 \\
\hline Staggered $S_{T} / S_{L}>2$ & $10^{3}-2 \times 10^{5}$ & 0.40 & 0.60 \\
\hline
\end{tabular}

Table 3.

fewer than 20 rows of tubes $\left(N_{L}<20\right)$ are present and is available in

This expression also takes into account the maximum turbulence and therefore $R e_{\max }$ is used, a variable based on the maximum fluid velocity. The maximum velocity occurs at the smallest area; transversally or diagonally between the tubes, according to Figure 5.

Table 2: Constants of equation (6) for airflow over a tube bank of 20 or more rows [41] - excerpt 
$\begin{array}{lll}2 \times 10^{5}-2 \times 10^{6} & 0.022 & 0.84\end{array}$

Table 3: Correction factor $C_{2}$ of equation (6) for $N_{L}<20$ [41]

\begin{tabular}{lccccccccc}
$N_{L}$ & 1 & 2 & 3 & 4 & 5 & 6 & 7 & 8 & 9 \\
\hline Aligned & 0.64 & 0.80 & 0.87 & 0.9 & 0.92 & 0.94 & 0.96 & 0.98 & 0.99 \\
Staggered & 0.68 & 0.75 & 0.83 & 0.89 & 0.92 & 0.95 & 0.97 & 0.98 & 0.99
\end{tabular}

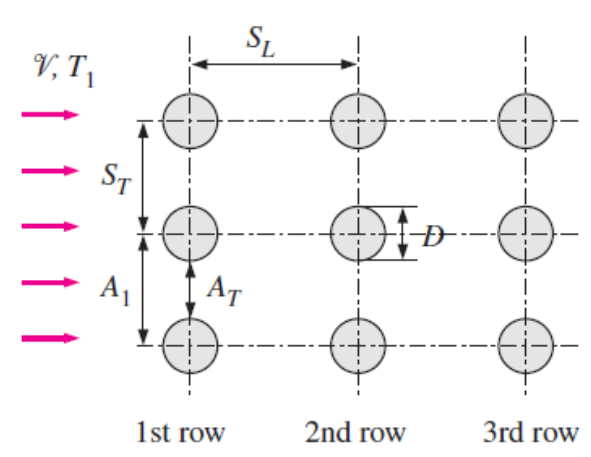

(a) In-line

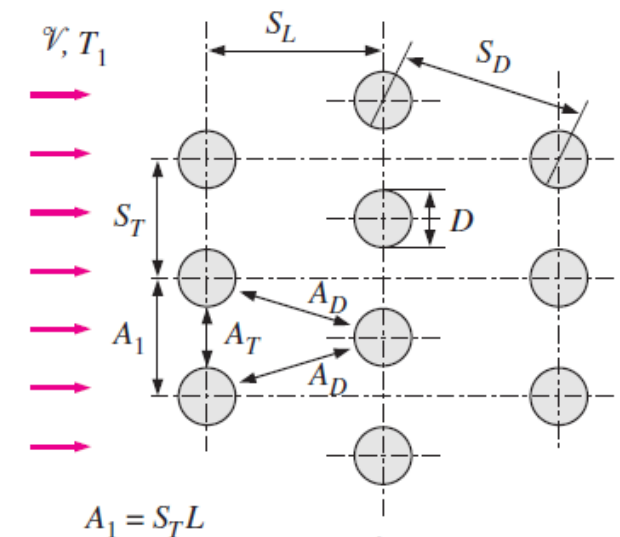

(b) Staggered

$A_{T}=\left(S_{T}-D\right) L$
$A_{D}=\left(S_{D}-D\right) L$

Figure 5: Tube arrangements in a bank (a) aligned and (b) staggered [42]

\subsection{Thermal resistance from vapour pressure drop}

The thermal resistance from vapour pressure drop $\left(R_{\text {in }}\right.$ in Figure 3$)$ changes as the vapour pressure decreases as it flows from the evaporator to the condenser section. The expression used for the vapour pressure drop is that of Faghri [43] and takes the form:

$$
R_{v}=\frac{8 R_{g} \mu_{v} T_{v}^{2}}{\pi h_{f g}^{2} P_{v} \rho_{v}}\left[\frac{\left(L_{e}+L_{c}\right) / 2+L_{a}}{r_{i}^{4}}\right]
$$

Where $R_{g}, h_{f g}, L_{a}, T_{v}, P_{v}, \mu_{v}$ and $\rho_{v}$ are the specific gas constant, latent heat of vaporisation of the working fluid, adiabatic section length and temperature, pressure, dynamic viscosity and density of the vapour phase, respectfully. The vapour temperature is the average temperature between the evaporator and condenser section temperatures and the vapour pressure is the saturation pressure correspondent to the vapour temperature.

\subsection{Boiling heat transfer}

Boiling regimes are dependent on the temperature difference between the bulk temperature of the fluid and the heating wall. In addition, evaporation on a pool of liquid is different from evaporation of a liquid film. The thermosyphons under study were all engineered to work in the nucleate pool boiling regime. The expression chosen to predict the heat transfer in nucleate pool boiling is that of Rohsenow \& Hartnett [44], found to be the most comprehensive correlation as it holds remarkably well and has been reported by many researchers in the literature; Reay \& Kew [7], Hagens et al. [13], Mroué et al. [37] and Ramos et al. [45] reported the use of Rohsenow \& Hartnett's expression [46] to predict the heat transfer from nucleate pool boiling in thermosyphons. Rohsenow \& Hartnett's expression for nucleate pool boiling has the form shown in equation (8).

The subscript $l$ refers to the liquid phase and $v$ to the gas phase as during boiling there is a mix of both. The coefficient $c_{s f}$ and the exponent $n$ depend on the surface-liquid combination. The thermosyphons under investigation are composed of carbon steel and filled with water having the following vales of $c_{s f}=0.006$ and $n=1.0$ [46]. 


$$
q^{n}=\mu_{l} h_{f g}\left[\frac{g\left(\rho_{l}-\rho_{v}\right)}{\sigma}\right]^{1 / 2}\left(\frac{c_{p l l}\left(T_{s a t}-T_{s}\right)}{c_{s f} h_{f g} P r_{l}{ }^{n}}\right)^{3} \quad\left(W / m^{2}\right)
$$

The thermal resistance offered by the boiling process may be found by first converting the heat flux to heat transfer rate by multiplying it with the heat transfer area and then divide the difference in temperature by this value, as shown in equation (9):

$$
R_{\text {bipool }}=\frac{T_{s}-T_{\text {sat }}}{q^{n} A_{\text {einside }}} \quad(K / W)
$$

It is important to note that the temperature of saturation of the fluid $\left(T_{\text {sat }}\right)$ and the Temperature of the boundary $\left(T_{s}\right)$ are required in order to solve the expressions related to the boiling and condensation of the working fluid. This is often resolved by employing thermocouples inside of the thermosyphon and on its surface.

\subsection{Condensation heat transfer}

Condensation is found to take place in the condenser section where the working fluid, upon coming into contact with the cooler walls, condenses and gives up its latent heat energy. For this heat transfer mechanism, the laminar condensation equation from Nusselt is used [47]:

$$
h_{\text {cond }}=0.943 \times\left(\frac{k_{l}^{3} \rho_{l}\left(\rho_{l}-\rho_{V}\right) g h_{f g}}{\mu_{l} \theta l}\right)^{1 / 4}
$$

Assuming that $\rho_{l} \gg \rho_{v}$ further simplifies the equation. Based on experiments, McAdams [48] also suggests a $20 \%$ increase to theoretical expressions due to the fact that experimental values are often larger, changing (10) into:

$$
h_{\text {cond }}=1.13 \times\left(\frac{k_{l}^{3} \rho_{l}^{2} g h_{f g}}{\mu_{l} \theta l}\right)^{1 / 4}
$$

\subsection{Predicting the performance of a thermosyphon-based heat exchanger}

The heat exchanger under study is equipped with 6 thermosyphons arranged in parallel as shown in the schematic represented by "Parallel" refers to their arrangement within the thermal network analogy. From a heat transfer perspective, the total thermal resistance for the heat exchanger as shown in Figure 6 would assume the six thermosyphons are in parallel with each other. This means that the overall thermal resistance would be smaller the more thermosyphons are included in the assembly. This is represented analytically in the following way:

$$
\frac{1}{R_{6 \text { thermosyphons (TS) }}}=\frac{1}{R_{i}}+\frac{1}{R_{i i}}+\frac{1}{R_{i i i}}+\frac{1}{R_{i v}}+\frac{1}{R_{v}}+\frac{1}{R_{v i}}
$$

The thermosyphons (TS) are assumed to have the same average internal thermal resistance thus simplifying the equation to:

$$
\frac{1}{R_{6 T S S}}=\frac{1}{R_{1 T S}} \times 6 \quad \therefore \quad R_{6 T S}=\frac{R_{1 T S}}{6}
$$

When looking at the larger picture as displayed in Figure 6, the overall thermal resistance for the thermosyphon-equipped heat exchanger $\left(R_{t h, T S H X}\right)$ may be found through the following expression:

$$
R_{t h, T S H}=R_{h_{i} e}+R_{6 T S s}+R_{h_{i} e}
$$


The subscript TS stands for thermosyphon, $e$ for evaporator, $c$ for condenser and $o$ for outer. Placing equation (13) into equation (14):

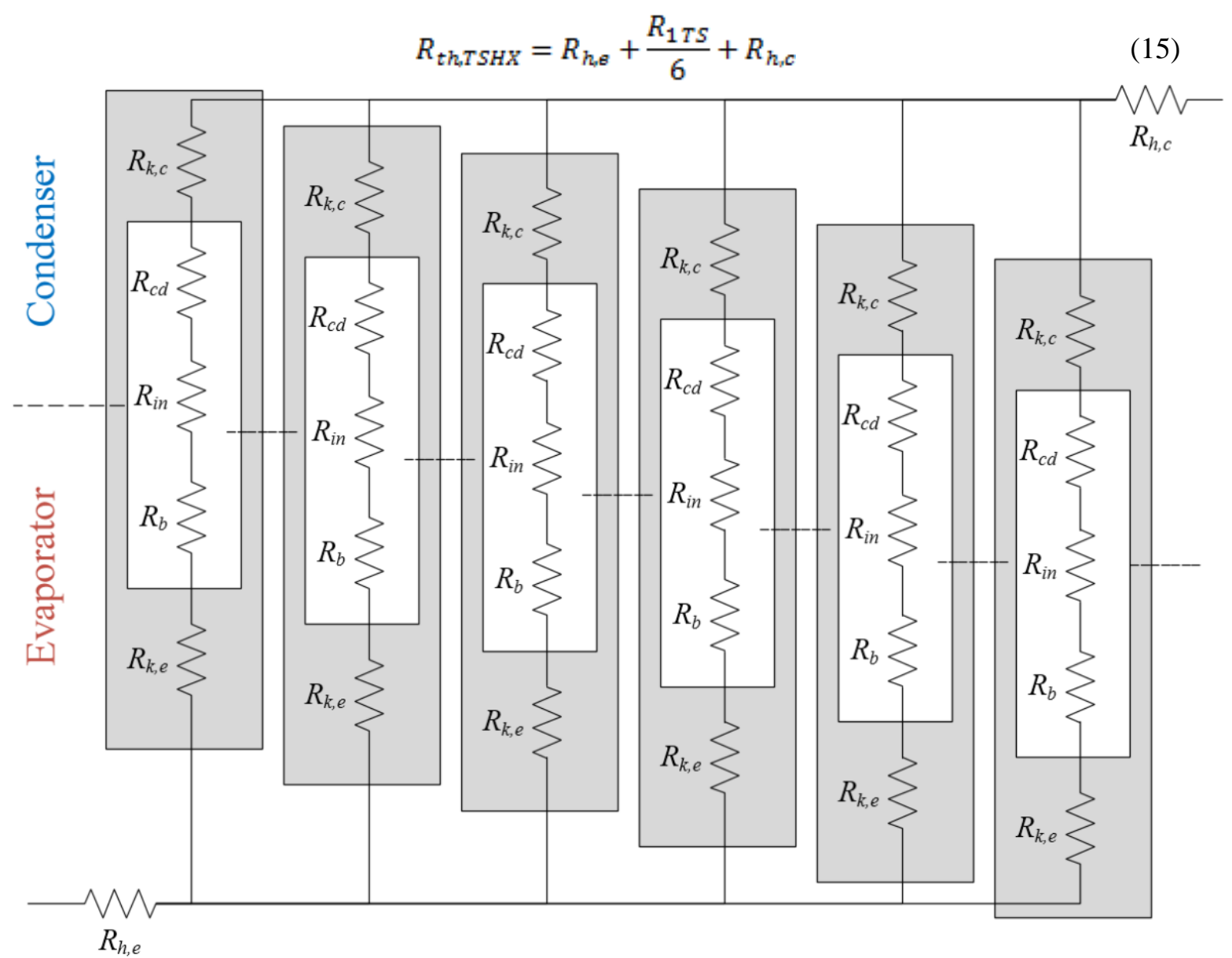

Figure 6: Schematic of the thermal resistances within the thermosyphons equipped in the heat exchanger

\subsubsection{Determination of the thermal conductivity of a single thermosyphon}

If the thermosyphon is assumed to be a solid super-conductor, this means that the total axial conductivity of a single thermosyphon may be taken as the axial conduction through a solid pipe:

$$
R_{1 T S}=\frac{L}{k A} \quad(K / W)
$$

Where $R_{I T S}$ is the overall thermal resistance of a single thermosyphon, $L$ is correlated to the length of the thermosyphon (in m), $k$ is the effective thermal conductivity for a single thermosyphon (in $\mathrm{W} / \mathrm{mK}$ ) and $A$ the cross sectional area $\left(\right.$ in $\mathrm{m}^{2}$ ). Re-arranging the equation for $k$ and considering the crosssectional area of a circle, equation (17) is obtained:

$$
k=\frac{L}{R_{1 t s} \pi r^{2}} \quad(W / m K)
$$

Equation (17) represents the thermal conductivity for a thermosyphon if it is assumed to be a solid super conductor. This value is used as a boundary condition in the CFD simulation.

\subsection{The effectiveness-NTU ( $\varepsilon$-NTU) prediction method applied to a TSHX}


$\varepsilon$-NTU stands for effectiveness-Number of Transfer Units and it is a method of predicting the performance of a heat exchanger. The number of transfer units is a dimensionless parameter widely used in heat exchanger analysis, generally defined as:

$$
\mathrm{NTU} \equiv \frac{U A}{C_{\min }}
$$

Where $U$ represents the overall heat transfer coefficient, $A$ the total heat transfer area and $C_{\min }$ the minimum heat capacity rate between the hot and cold flows. The heat capacity rate is a measure of the mass flow rate $(\dot{m}$ in $\mathrm{kg} / \mathrm{s})$ multiplied by the specific heat capacity $\left(c_{p}\right.$ in $\left.J / k g . K\right)$.

Effectiveness $(\varepsilon)$ is the ratio of the actual heat transfer rate to the maximum possible heat transfer rate; which would be achieved if the temperature of the outlet of the cold flow would equal the inlet temperature of the hot flow [26]:

$$
\varepsilon \equiv \frac{q_{a c t}}{q_{\max }} \equiv \frac{C_{c}\left(T_{h, i}-T_{h, o}\right)}{C_{\min }\left(T_{h, i}-T_{c, i}\right)} \quad \text { and } \quad \varepsilon=\frac{C_{c}\left(T_{c, o}-T_{c, i}\right)}{C_{\min }\left(T_{h, i}-T_{c, i}\right)}
$$

Where $C_{h}$ and $C_{c}$ represent the heat capacity of the hot and cold flows, respectively, and $C_{\min }$ the smallest between the two. By definition, effectiveness is dimensionless and must be valued between 0 and 1 ; theoretically only a heat exchanger of infinite length would be able to achieve an effectiveness of 1 .

The $\varepsilon$-NTU analysis of a heat exchanger equipped with thermosyphons may be done by separating it into two separate heat exchangers, the condenser and the evaporator, and consider them coupled by the thermosyphon working fluid $[6,24,38,43]$. The effectiveness of the evaporator and condenser sections of the heat exchanger is determined separately and is given by:

$$
\varepsilon_{e}=1-e^{\left(-N T U_{e}\right)} \text { and } \varepsilon_{c}=1-e^{\left(-N T U_{e}\right)}
$$

The number of transfer units for the evaporator and for the condenser is found through:

$$
\mathrm{NTU}_{e}=\frac{U_{e} A_{e}}{C_{e}} \text { and } \mathrm{NTU}_{c}=\frac{U_{c} A_{c}}{C_{c}}
$$

Where $A$ refers to the total heat transfer area in the respective row or stage, and $C_{e}$ and $C_{c}$ represent the average heat capacity of the shell-side fluid. The overall heat transfer coefficient $U$ must be found for each section using the thermal network analogy explained in a later chapter.

Faghri [43] defined the effectiveness of an individual thermosyphon to be related to the minimum and maximum values of effectiveness between the evaporator and the condenser sections:

$$
\varepsilon_{t s}=\left(\frac{1}{\varepsilon_{\min }}+\frac{C_{r}}{\varepsilon_{\max }}\right), \quad C_{r}=\frac{C_{\min }}{C_{\max }}
$$

Where $\varepsilon_{\min }$ and $\varepsilon_{\max }$ take the minimum and maximum values of $\varepsilon_{e}$ and $\varepsilon_{c} . C_{r}$ is the heat capacity ratio and $C_{\min }$ and $C_{\max }$ follow the same logic of the effectiveness, taking the minimum and maximum values of $C_{e}$ and $C_{c}$, respectively.

The effectiveness of a multistage heat exchanger in counter flow for an $n$ number of rows has been adapted from Incropera \& DeWitt [47] in order to apply to thermosyphon-equipped heat exchangers. 


$$
\begin{aligned}
& \varepsilon_{\theta, n}=\frac{\left(\frac{1-C_{r_{i},} \varepsilon_{e}}{1-\varepsilon_{\theta}}\right)^{n}-1}{\left(\frac{1-C_{r_{i},} \varepsilon_{e}}{1-\varepsilon_{e}}\right)^{n}-C_{r_{i} \theta}} \\
& \varepsilon_{c, n}=\frac{\left(\frac{1-C_{r_{i} c} \varepsilon_{c}}{1-\varepsilon_{c}}\right)^{n}-1}{\left(\frac{1-C_{r, c} \varepsilon_{c}}{1-\varepsilon_{c}}\right)^{n}-C_{r, c}}
\end{aligned}
$$

$C_{r}$ is the heat capacity ratio of the fluid streams on each side of the thermosyphon; the ratio between the heat capacity rate of the shell-side fluid to the heat capacity rate of the thermosyphon's working fluid. However, since the working fluid is at constant temperature, its specific heat and capacity rate is effectively infinite, making the variables $C_{r, e}$ and $C_{r, c}$ equal to zero [39]. Equations (23) and (24) are then simplified into the forms seen in equation (25) and (26), respectively:

$$
\begin{aligned}
& \varepsilon_{e, n}=1-\left(1-\varepsilon_{e}\right)^{n} \\
& \varepsilon_{c, n}=1-\left(1-\varepsilon_{c}\right)^{n}
\end{aligned}
$$

The overall effectiveness depends on which fluid side has the largest heat capacity; if the heat capacitance of the evaporator side fluid is the largest; $C_{e}>C_{c}$ :

$$
\varepsilon_{t}=\left(\frac{1}{\varepsilon_{c, n}}+\frac{C_{d} / C_{e}}{\varepsilon_{e, n}}\right)^{-1}
$$

On the other hand, if $C_{c}>C_{e}$ :

$$
\varepsilon_{t}=\left(\frac{1}{\varepsilon_{\theta, n}}+\frac{C_{e} / C_{c}}{\varepsilon_{\varepsilon_{e}, n}}\right)^{-1}
$$

Using the overall effectiveness of the heat exchanger, the outlet temperatures for the evaporator and the condenser can be predicted from:

$$
\begin{aligned}
& T_{h, \text { out }}=T_{h, \text { in }}-\varepsilon_{t} \frac{C_{\text {min }}}{C_{e}}\left(T_{h, \text { in }}-T_{c, \text { in }}\right) \\
& T_{c, \text { out }}=T_{c, \text { in }}+\varepsilon_{t} \frac{C_{\text {min }}}{C_{c}}\left(T_{h, \text { in }}-T_{c, \text { in }}\right)
\end{aligned}
$$

\section{$3 \quad$ Experimental set up}

This chapter describes the physical design of the experimental rig, the working conditions of the heat exchanger, including the inlet temperatures and flow rates of both shell sides, as well as the control apparatus used.

\subsection{Design of test rig}

The experimental rig consisted of a heat exchanger equipped with six thermosyphons in a cross-flow arrangement. The design of the rig was based on a real heat exchanger used by the partner company, built with a modular design in mind in order to allow further investigation of different flow configurations and boiling regimes.

The test rig was equipped with six thermosyphons vertically arranged in two staggered rows. The thermosyphon tubes were made of carbon steel measuring $2 \mathrm{~m}$ in length and with a diameter of $28 \mathrm{~mm}$. 
The surrounding wall had an average thickness of $2.5 \mathrm{~mm}$. The working fluid was distilled water and the filling ratio was $100 \%$ (of the evaporator section), which roughly translates into $0.7 \mathrm{~m}$ in height from the bottom of the thermosyphon. All tubes were chemically treated before insertion of water to avoid corrosion.

As can be seen in Figure 7, the condenser section occupied the top $0.2 \mathrm{~m}$ of the thermosyphons and the evaporator section the lower $0.6 \mathrm{~m}$. The remaining $1.2 \mathrm{~m}$ were kept fully insulated as they served the adiabatic section of the heat pipe. Both the evaporator and the condenser were separated from the adiabatic section by a $10 \mathrm{~mm}$-thick division plate in order to prevent leaks.
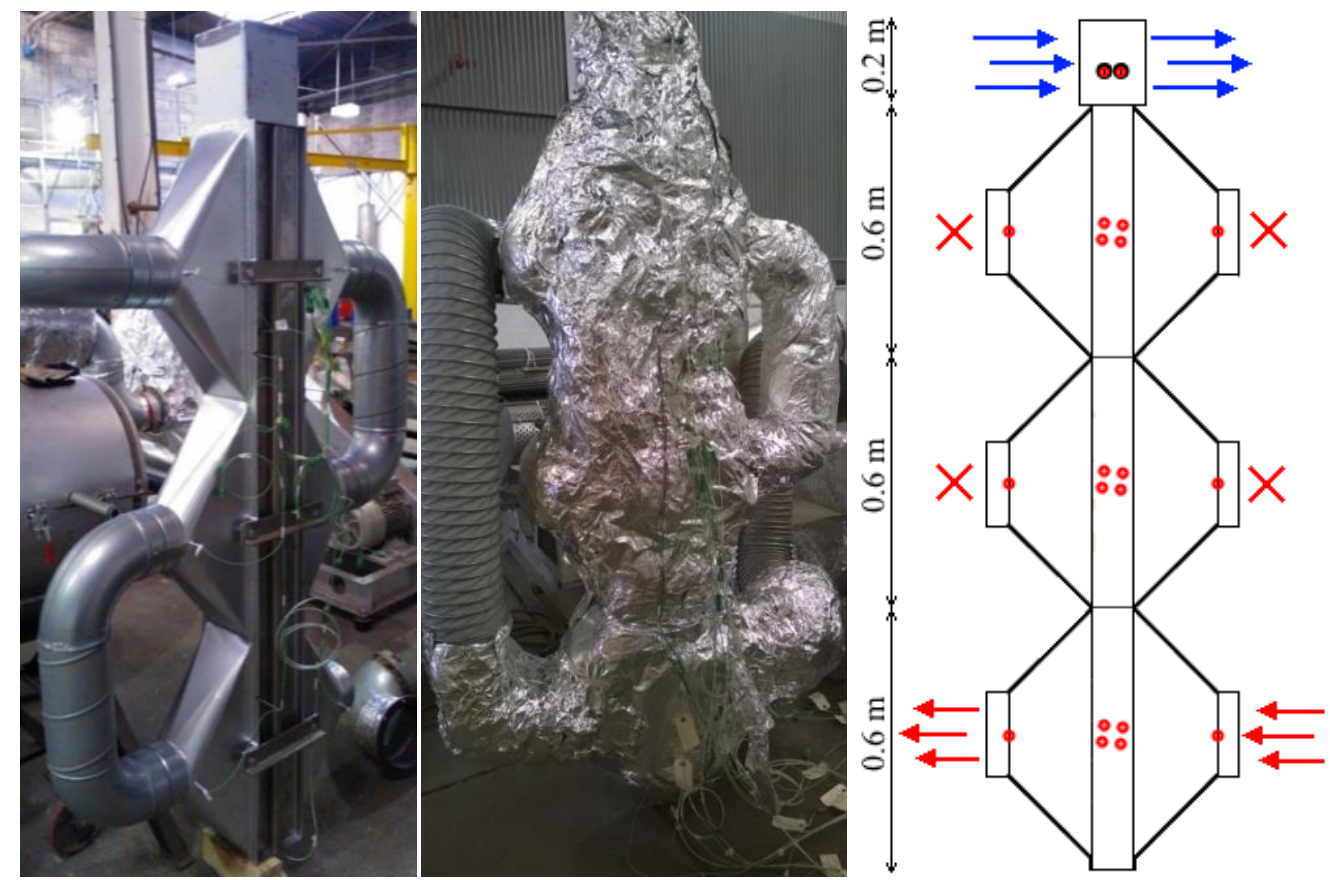

\section{Condenser} section

\section{Adiabatic section}

Figure 7: Experimental apparatus of the heat exchanger in cross flow

From left to right: the heat exchanger before installation; the heat exchanger after being thermally insulated; representative schematic of the thermosyphon heat exchanger and the size of its respective sections.

\subsection{Experiment design}

The experimental rig was divided between two circuits; a closed air circuit - the heat source - and an open water circuit - the heat sink -, both included in the schematic represented in Figure 8. The hot air circuit consisted of a closed air loop equipped by a fan and a heater. The flow was directed to pass through the fan and the heater and then to enter the heat exchanger. After leaving the heat exchanger, it was sucked into the fan once again, repeating the cycle. The fan frequency ranged between 10 and $50 \mathrm{~Hz}$ in $10 \mathrm{~Hz}$ increments. The mass flow rate was controlled by an analogue pitot tube installed at the inlet of the evaporator section and ranged between an average 0.05 and $0.14 \mathrm{~kg} / \mathrm{s}$ in 0.03 increments.

The heater power could be regulated according to a desired temperature thanks to a feedback loop. The feedback loop controlled the heater power through a thermocouple located at the outlet of the heat exchanger. The temperature of the air varied between 100 and $300^{\circ} \mathrm{C}$ in $50^{\circ} \mathrm{C}$ increments. The higher temperatures correspond to the normal working conditions of the heat exchangers encountered in waste heat recovery [12]. The lower temperatures were employed to test the lower operating limits of the thermosyphons. 


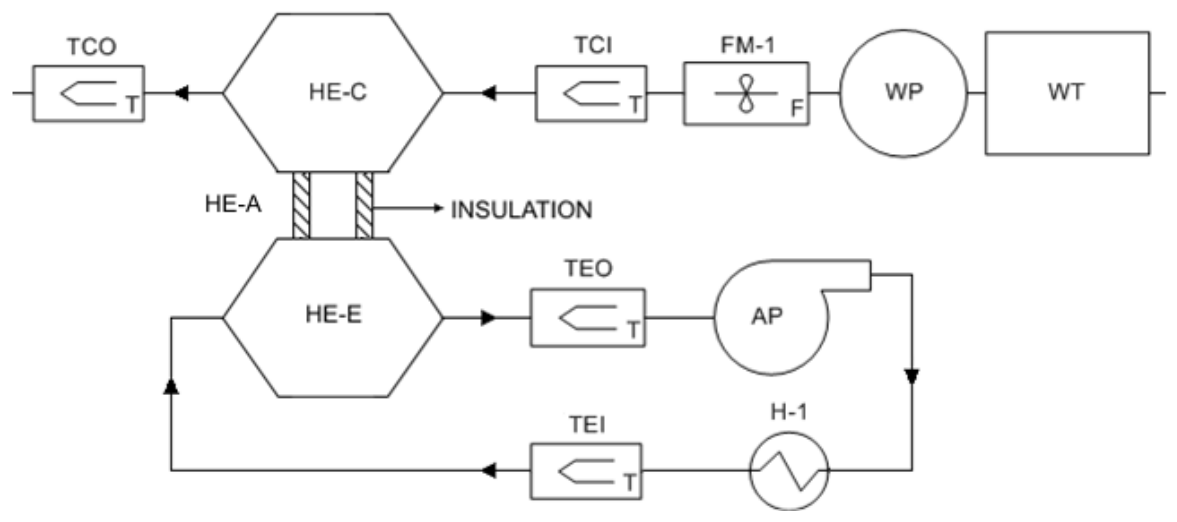

Figure 8: Schematic of the single pass test setup

Description: TCI/TCO - Thermocouples at inlet/outlet of condenser; HE-C/E/A - Thermosyphon heat exchanger Condenser/Evaporator/Adiabatic section; FM-1 - Turbine Flow Meter; WP - Water pump; WT - Water tank; TEI/TEO - Thermocouple at inlet/outlet of evaporator; AP - Air Pump; H-1 - Air Heater.

The cold water circuit consisted of an open loop and included a water tank to help regulate the inlet flow rate into the pump as can be seen in Figure 8. The mass flow rate of water was kept constant throughout the test at $0.08 \mathrm{~kg} / \mathrm{s}$ and at an average temperature of $10^{\circ} \mathrm{C}$. After leaving the water tank, the water was pumped through the heat exchanger. After flowing through the heat exchanger, the warmed-up water would freely flow into another process. The flow inside the condenser section followed a U-shaped path as depicted in Figure 4.

\subsection{Gathering and Processing of Data}

20 k-type thermocouples were placed at specific locations in the heat exchanger to measure the temperature of the flows and the working temperatures of the thermosyphons. The thermocouples were placed in key sections, such as the inlet and outlet of both the evaporator and condenser sections, on the surface of each "corner" thermosyphon (numbered 1 to 4 in Figure 4), on the adiabatic section, and within the thermosyphons. The thermocouple placements are marked in red in the simplified schematic of Figure 7.

Four thermocouples were placed on the surface of each "corner" thermosyphon: one in the evaporator section, two in the adiabatic section and one in the condenser section. These thermocouples were brazed into the surface of the thermosyphon at $1 \mathrm{~mm}$ depth. Two brazed thermocouples are shown in Figure 9.

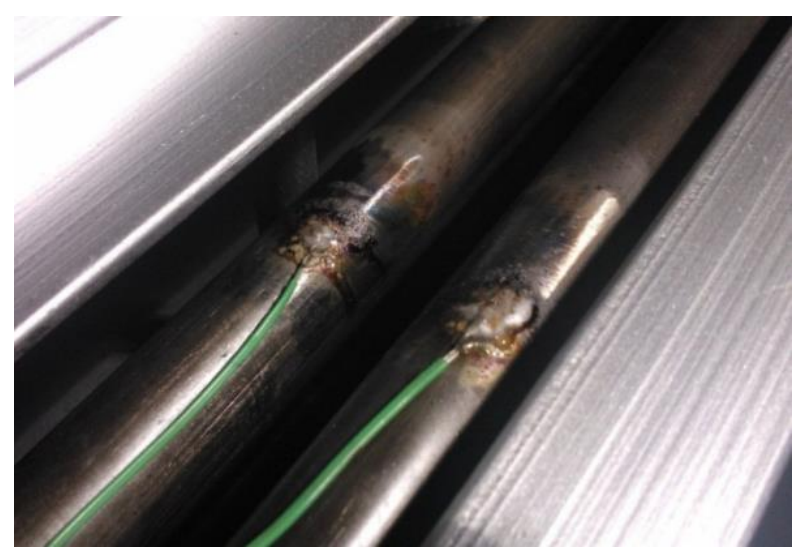

Figure 9: The brazed thermocouples on the surface of the thermosyphon

The experimental procedure was the same in all the tests and it is described as follows: 
1. The cold water was allowed to run in the cold water circuit.

2. The air was then bled from the condenser section through the bleed valve located at the top.

3. After the condenser section was filled with water, the heater was turned on and the hot air flow was flowing in the evaporator section.

4. The temperature was set to $300^{\circ} \mathrm{C}$. Data for this temperature setting would be recorded for a fan rate of 10,20,30, 40 and $50 \mathrm{~Hz}$. The same flow rates were then tested for 250, 200, 150, 100 and $50^{\circ} \mathrm{C}$.

5. Data were recorded and collected for each setting every 10 minutes at steady state conditions.

A total of 30 tests were conducted, one for each different inlet condition.

\section{The CFD model}

A 3 dimensional computational model was run in parallel with the experimental tests conducted on the heat exchanger. In this simplified model the thermosyphons were modelled as super-conductors whose thermal conductivity had been deduced according to the inlet conditions using the analytical method explained in chapter 2 .

\subsection{Assumptions}

The following assumptions were made prior to running the simulation:

a) Constant mass flow rate across the heat exchanger in both flow sides

b) Neglectable axial heat transfer from conduction across the thermosyphon wall

c) No heat transfer across the walls of heat exchanger

d) No heat transfer at the adiabatic section of the thermosyphon

e) Constant inlet mass flow rate across inlet area

f) Same thermal conductivity for all the thermosyphons

g) The thermosyphons were assumed to be solid superconductors

\subsection{Methodology}

ANSYS Fluent was the computational fluid dynamics (CFD) software used to simulate the heat flow within the heat exchanger. The model was created with the purpose of assessing the potential of simulating heat pipes as solids rods of constant conductivity for the modelling of future heat exchangers. The realizable k-epsilon turbulence model $(k-\varepsilon)$ was used in each of the simulations as it is found to be more accurate at higher Reynolds number and smaller pressure gradients $[49,50]$, which is the case in this particular experimental test range. A coupled pressure-based solver is also recommended as it is more efficient in steady-state simulations and offers better results for singlephase fluid flow $[45,51]$.

The thermosyphons were modelled as solid objects using the value for thermal conductivity achieved from the method described in chapter 3. For the fluid properties, Fluent's own standard tables of substance properties were used to determine the characteristics of the fluids simulated (water in the condenser and air in the evaporator).

\subsection{Mesh selection}

The mesh selection was done by running the same simulation with different mesh sizes and comparing the accuracy of the results. A mesh was deemed "good" if the maximum skewness is lower than 0.7 for hexahedron and tetrahedrons and 0.8 for triangular elements [20]. A comparison of the results is available in Table 4 and can be observed in Figure 10.

Table 4: Mesh Dependency.

\begin{tabular}{|ccccc|}
\hline Level & No of Cells & Type of cells & Skewness & Time per iter. \\
Coarse & $1,408,658$ & Hex + Tetra & avg: 26\%, stdev: $16 \%$ & $2-10 \mathrm{~s}$ \\
Medium & $2,291,364$ & Hex + Tetra & avg: $21 \%$, stdev: $13 \%$ & $7-15 \mathrm{~s}$ \\
Fine & $3,099,230$ & Hex + Tetra & avg: 21\%, stdev: $13 \%$ & $25-50 \mathrm{~s}$ \\
\hline
\end{tabular}




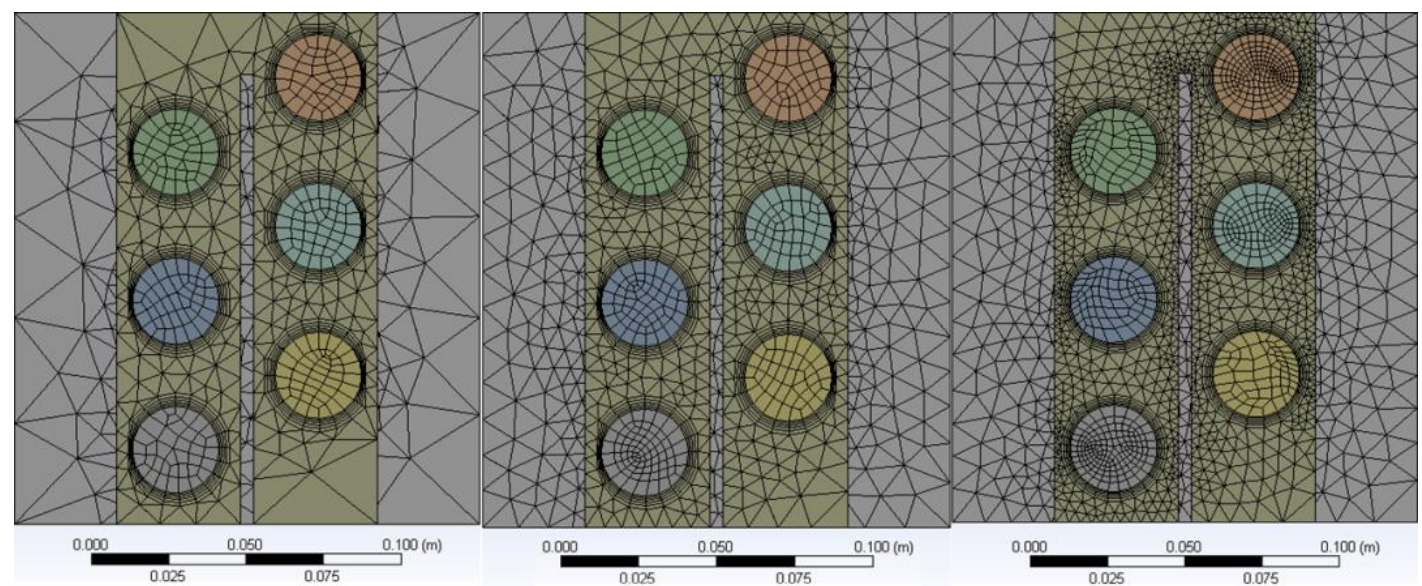

Figure 10: Comparison between the three different meshes - Coarse, Medium and Fine

It was found that a fine mesh would take 3 times longer to converge (on average) and the results would not be significantly more accurate $( \pm 0.8 \%)$; therefore the medium mesh was used in all the tests run in Fluent.

The relaxation factors were set at $1 \mathrm{e}-6$ and the test allowed to run until no change was observed in the scaled residuals.

\subsection{Boundary Conditions}

The boundary conditions used in the CFD to describe the inlet and outlet of the heat exchanger model are displayed in Table 5. The CFD model was run several times for each different inlet condition to reduce the variance of the results.

Table 5: Boundary Conditions

\begin{tabular}{|llcc|}
\hline & Type & Mass flow rate $(\mathbf{k g} / \mathbf{s})$ & Temperature $\left({ }^{\circ} \mathbf{C}\right)$ \\
Evaporator Inlet & Mass Flow Inlet & 0.05 to 0.17 at 0.03 & 50 to 300 at 50 \\
& & intervals & intervals \\
Evaporator Outlet & Mass Flow Inlet & - & Desired Output \\
Condenser Inlet & Mass Flow Inlet & Constant 0.0715 & Constant 10.0 \pm 0.3 \\
Condenser Outlet & Mass Flow Inlet & - & Desired Output \\
\hline
\end{tabular}

The boundary conditions used to simulate the inlets and outlets were of type "mass flow inlet", with the outlets having the opposite direction. This assumption is valid for both circuits: the air circuit consisted of a closed system, as observed in Figure 8, so it was being "pulled" out of the evaporator outlet at the same rate it was pushed into the evaporator inlet.

At normal atmospheric conditions, water is incompressible. Since the condenser section had been completely purged of air prior to the start of any test, the assumption is that the mass flow rate of water at the exit of the condenser must be the same as the mass flow rate of water flowing into the condenser.

All the walls of the heat exchanger were insulated during the experimental test and were thus assumed to be adiabatic in the CFD simulation. The only contact between the hot and cold flows is made through the thermosyphons.

The thermosyphons were modelled not as two-phase devices, but as solid bars. The thermal conductivity of the tubes was predicted using the thermal network analogy described in chapter 2.

\section{$5 \quad$ Results and discussion}


This chapter presents the main results and includes a comparison between the experimental, theoretical and CFD results. First the results from the experimental tests are presented and then a comparison of the results obtained from the CFD and the numerical predictions is included.

\subsection{Experimental Results}

This section outlines the results for the experiment with six thermosyphons. Air was used as the evaporator-side fluid and water as the condenser-side fluid. The mass flow rate of air varied between 0.05 and $0.17 \mathrm{~kg} / \mathrm{s}$ and the inlet air temperature varied between 50 and $300^{\circ} \mathrm{C}$. On the condenser side, the inlet temperature and the mass flow rate of water were both kept constant at approximately $7^{\circ} \mathrm{C}$ and $0.08 \mathrm{~kg} / \mathrm{s}$ respectively.

Figure 11 displays the temperature distribution within the heat exchanger for inlet temperatures of 50, $100,150,200,250$ and $300^{\circ} \mathrm{C}$. The temperatures were logged from five different locations; namely at the inlet and outlet of the evaporator $\left(T_{\text {ein }}, T_{\text {eout }}\right)$ and condenser sections $\left(T_{\text {cin }}, T_{\text {cout }}\right)$ and inside the thermosyphons $\left(T_{\text {pipe }}\right)$.

It can be seen that the trend is for the difference in temperature across the evaporator section to decrease as the mass flow rate of the incoming hot air increases. In the condenser section the difference in temperature increases with increasing mass flow rate on the evaporator side. From a thermodynamic perspective this is a logical outcome as the increasing mass flow rate of air into the evaporator increases the heat transfer coefficient which in turn increases the heat flow into the thermosyphon thus resulting in more heat being transferred to the water on the condenser section.



Air mass flow rate $(\mathrm{kg} / \mathbf{s})$

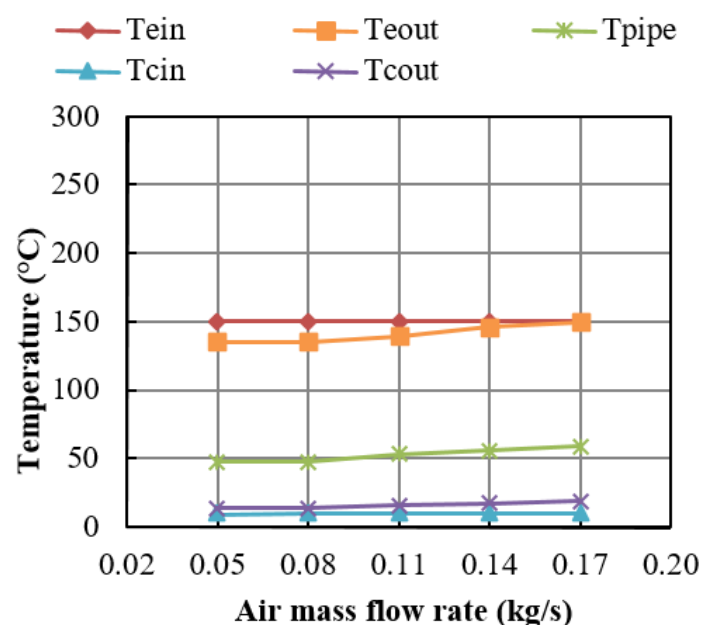

Air mass flow rate (kg/s)
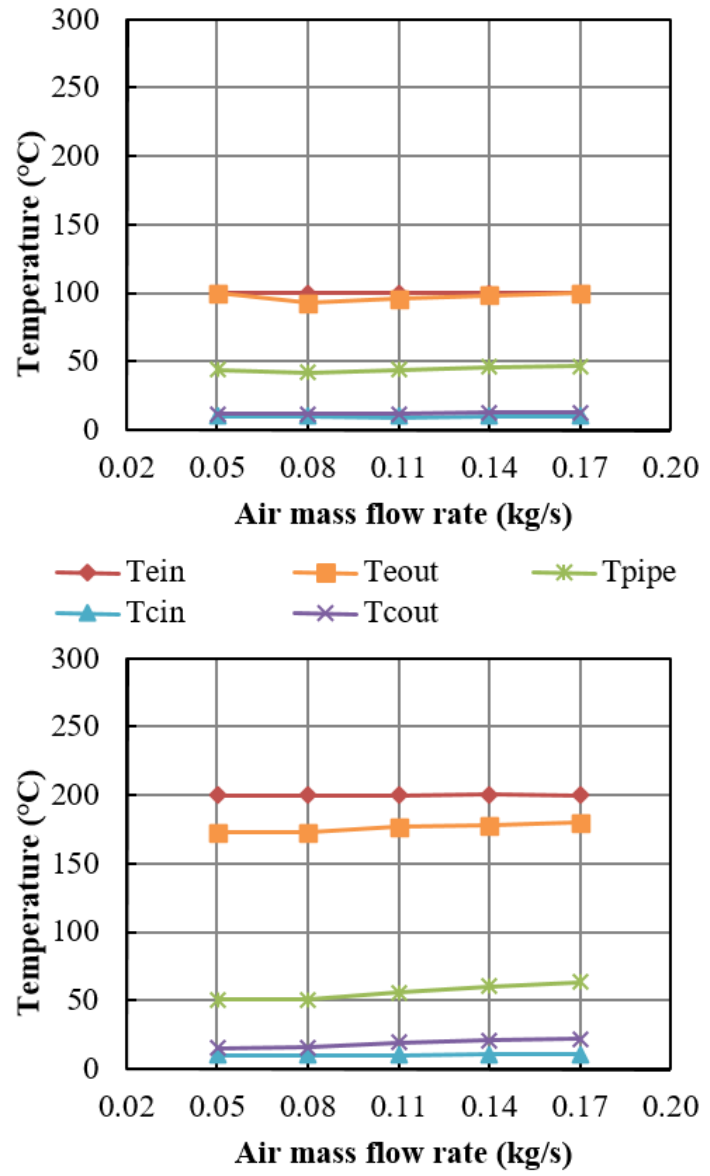


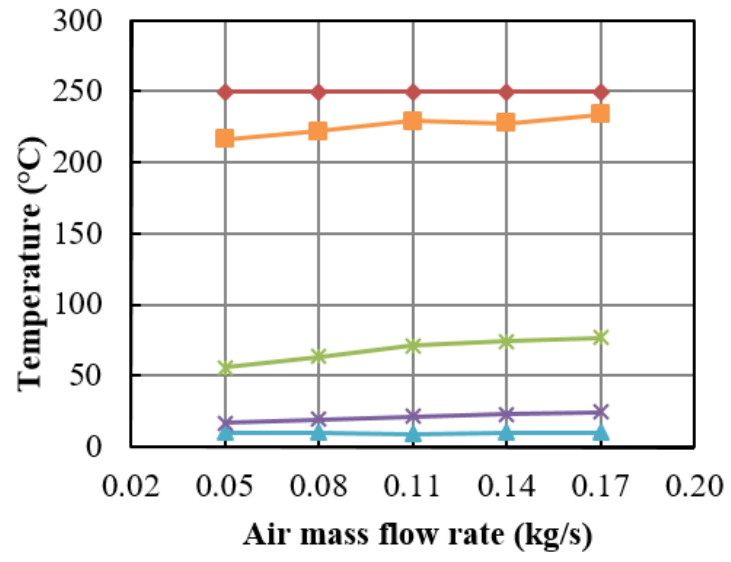

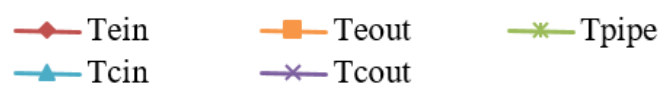

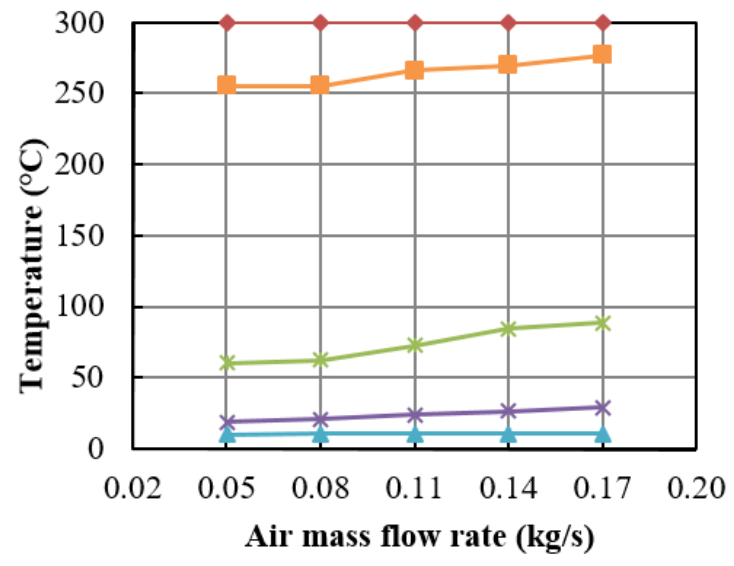

$\because$ Tein
—-Tpipe

Figure 11: Temperature distribution within the heat exchanger for inlet air temperatures ranging from 50 to $300{ }^{\circ} \mathrm{C}$

From the experimental results, a regular pattern emerged between the thermosyphon working temperature and the overall difference in temperature across the entire heat exchanger $\left(T_{e, a v g}-T_{c, a v g}\right)$. A plot of the average working temperature of the thermosyphons against the overall difference in temperature between the evaporator and the condenser section for the range of mass flow rates tested is displayed in Figure 12. Data from the trend lines, shown in their respective colour, allowed the creation of equation (31) an expression able to predict the average working temperature of the thermosyphons for each different working conditions.

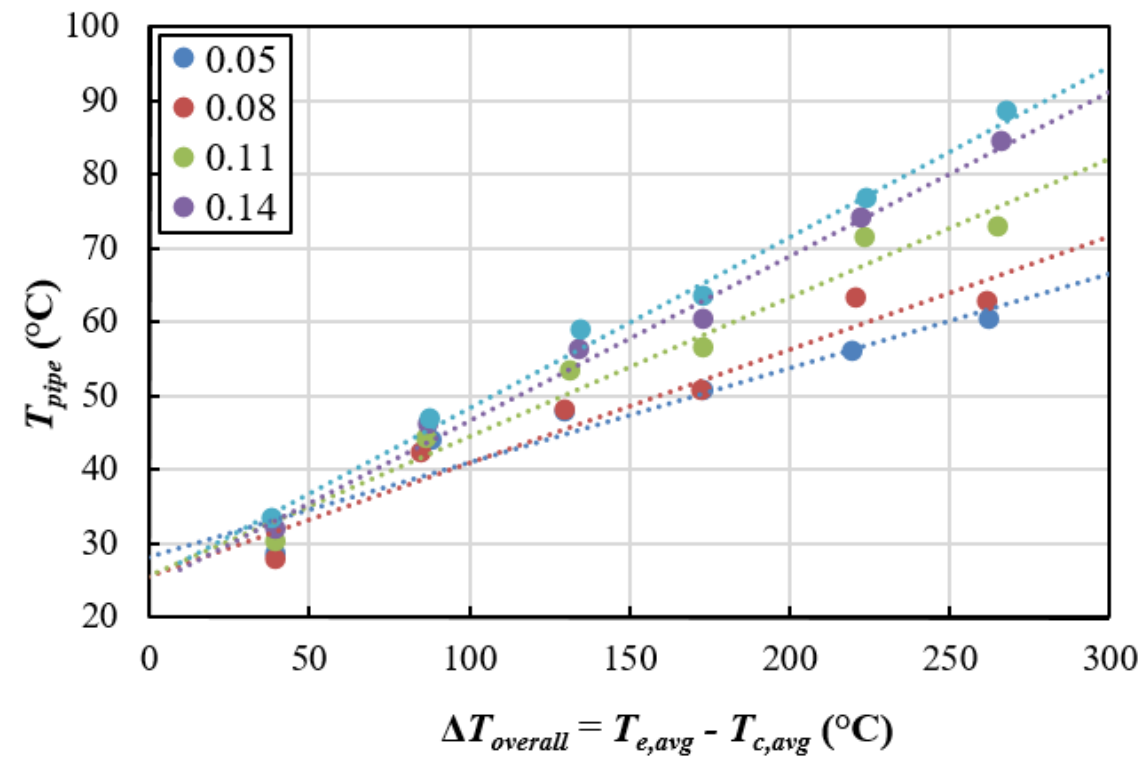

Figure 12: Average working temperature of the thermosyphons for different overall $\Delta \mathrm{T}$ at different mass flow rates

$$
T_{\text {pipe }}=0.589 \dot{m}^{0.5146}\left(\Delta T_{\text {overall }}-40\right)+32 \quad\left({ }^{\circ} \mathrm{C}\right)
$$

Equation (31) is a correlation that allows the prediction of the average working temperature of the thermosyphons according to the mass flow rate of air on the evaporator side and the difference in temperature across the entire heat exchanger. The expression only applies to the heat exchanger under 
study with constant temperature and mass flow rate on the condenser side. The output of the applied correlation to the inlet conditions of the heat exchanger is presented in Figure 13 which seems to follow the tendency of Figure 12 with reasonable accuracy.

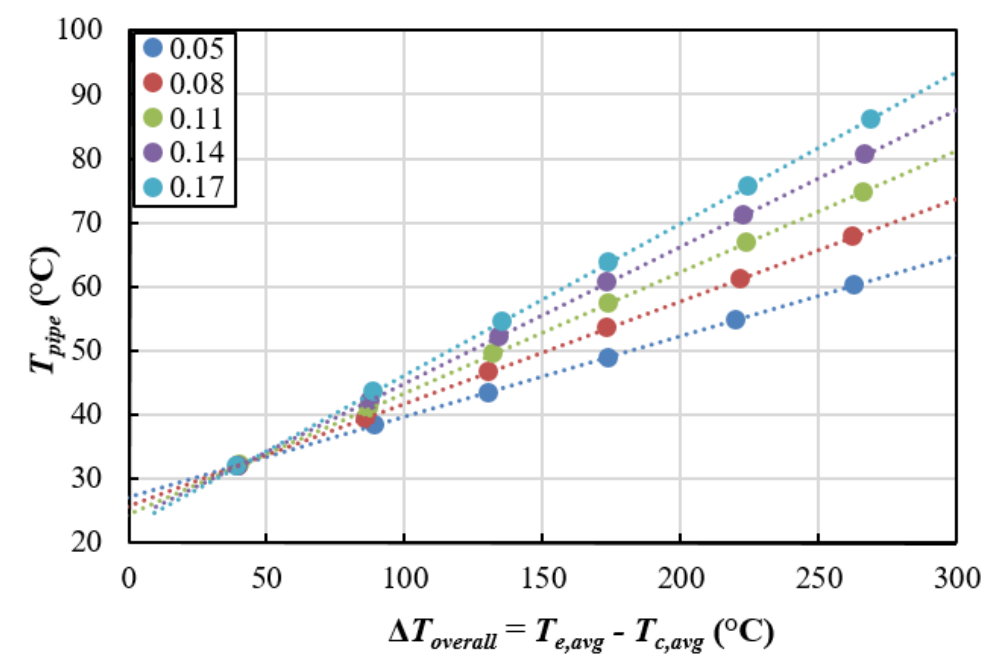

Figure 13: Predicted average working temperature of the thermosyphons for different overall $\Delta \mathrm{T}$ at different mass flow rates

The heat transfer rate was found to be directly proportional to the inlet air mass flow rate and to the temperature of the flow at the inlet, as can be seen in Figure 14. The profile of the lines indicate that if a higher mass flow rate was provided, the heat exchanger would be capable of transporting that much more heat, however, the lines also start to become more flat as the mass flow rate increases. The average maximum duty according to the data gathered was $900 \mathrm{~W}$ maximum heat flux per heat pipe.

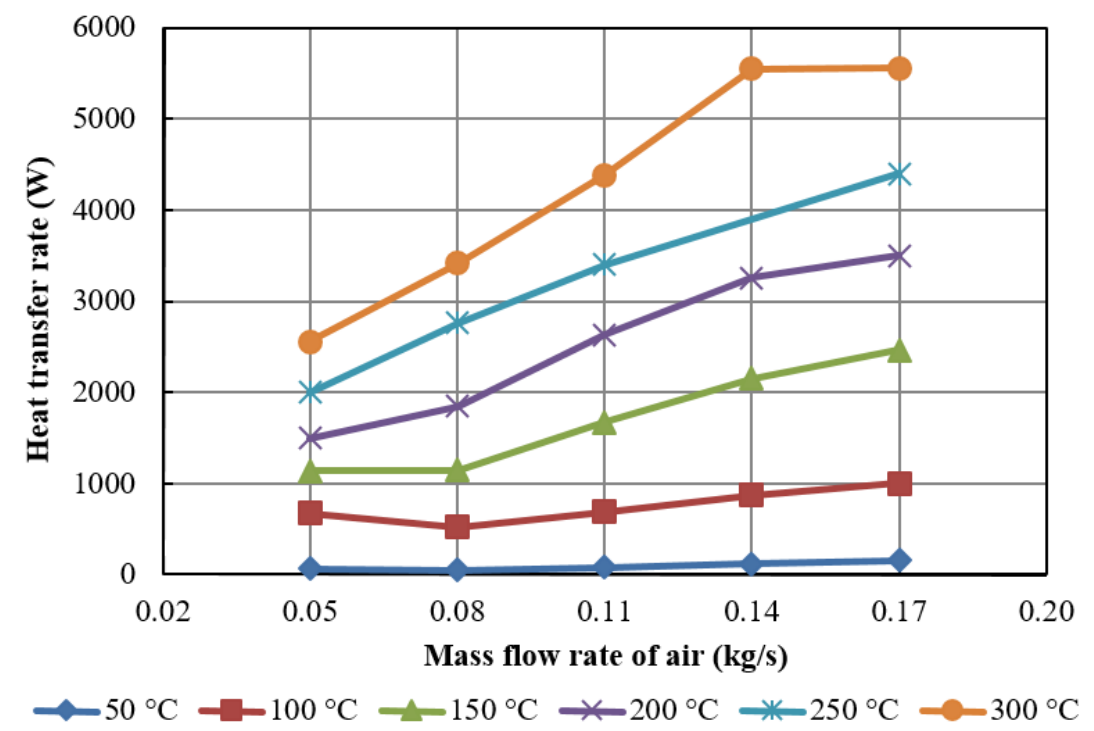

Figure 14: Total heat transfer rate of the heat exchanger

The thermal resistance from equation Error! Reference source not found. was also plotted against the overall heat transfer rate as shown in Figure 15. A higher difference in temperature produced a lower overall thermal resistance in the heat exchanger due to the more stable boiling regime inside the thermosyphon. Once again, the results obtained at $50^{\circ} \mathrm{C}$ are the oddball with a thermal resistance 
higher than $0.2 \mathrm{~K} / \mathrm{W}$. The other results have a lower value ranging between 0.05 and $0.1 \mathrm{~K} / \mathrm{W}$. The plot clearly shows the thermal resistance is inversely proportional to the overall heat transfer coefficient.

An uncertainty study was conducted in order to find the error propagation from the measurement instruments used in the experimental rig. It was observed that for all the tests, the uncertainty when determining the $Q_{\text {out }}$ is lower than $10 \%$, which is an acceptable figure for engineering applications.

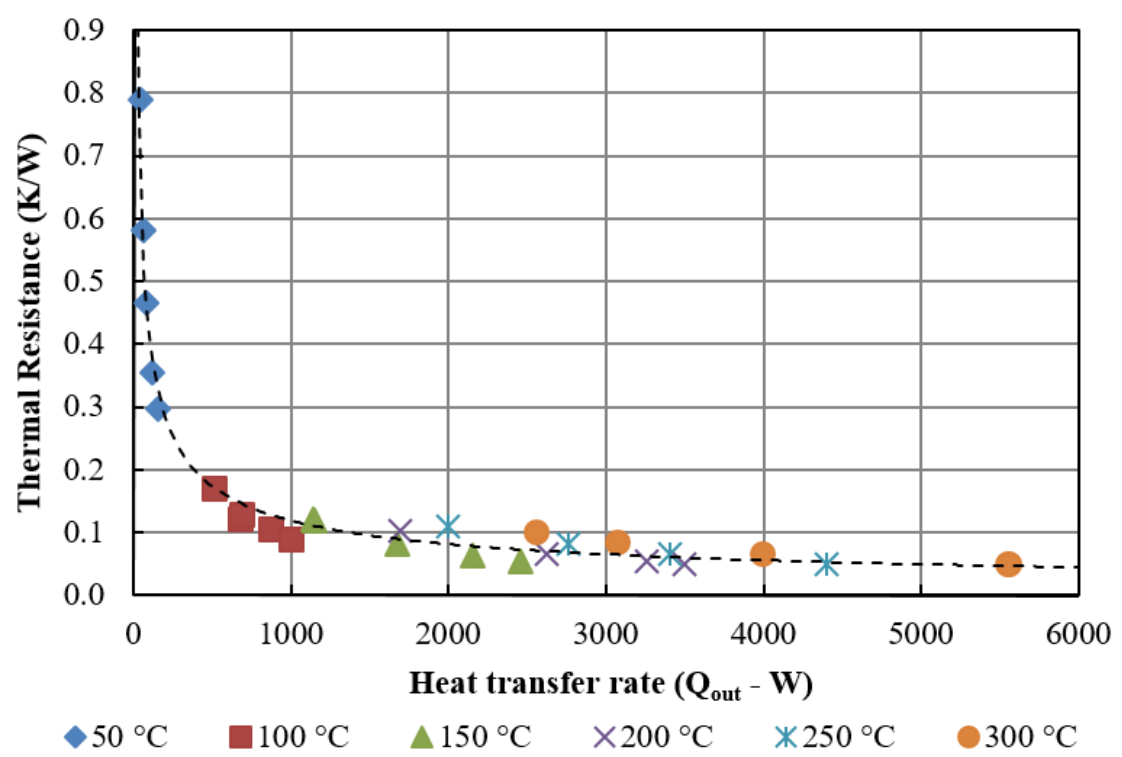

Figure 15: Relation between $Q_{\text {out }}$ and the overall thermal resistance

\subsection{Effectiveness}

Effectiveness is a variable that is an integral part of the Effectiveness-Number of Transfer Units ( $\varepsilon$ NTU) method and is a measure of a heat exchanger's heat transfer potential. The effectiveness is a rate of the actual heat transfer of a heat exchanger to the maximum possible heat transfer rate.

Figure 16 represents a plot of the effectiveness of the heat exchanger against the mass flow rate of incoming air. A downward trend is observed in all of the results, as with the increased mass flow rate, the temperature difference between the inlet and the outlet of the condenser section is reduced, reducing the overall effectiveness. The plot is in agreement with Jouhara and Merchant [26] as higher inlet temperatures result in higher effectiveness.

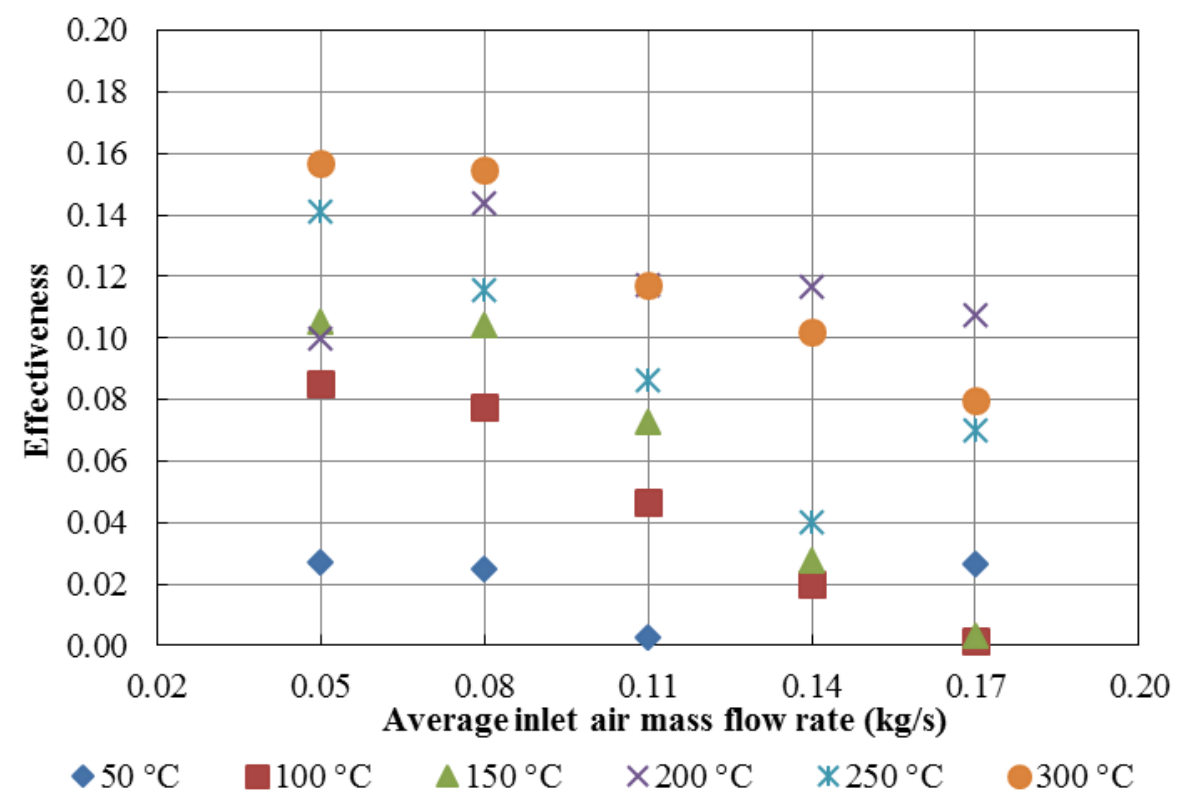


Figure 16: Effectiveness of the heat exchanger

The effectiveness was also plotted against the Number of Transfer Units (NTU) and it can be concluded that there is a quasi-linear relation in agreement with the literature for this type of plots [47, 42] as seen in Figure 17. The heat exchanger under study had a small area of exposure and was incapable of transferring more than 0.2 transfer units, however, the trend shown in Figure 17 displays a linear increase. The effectiveness of the heat exchanger will increase at a rate of 9:10 to the number of transfer units until an effectiveness of 1 which at the current rate could be found at $1.2 \mathrm{NTU}$.

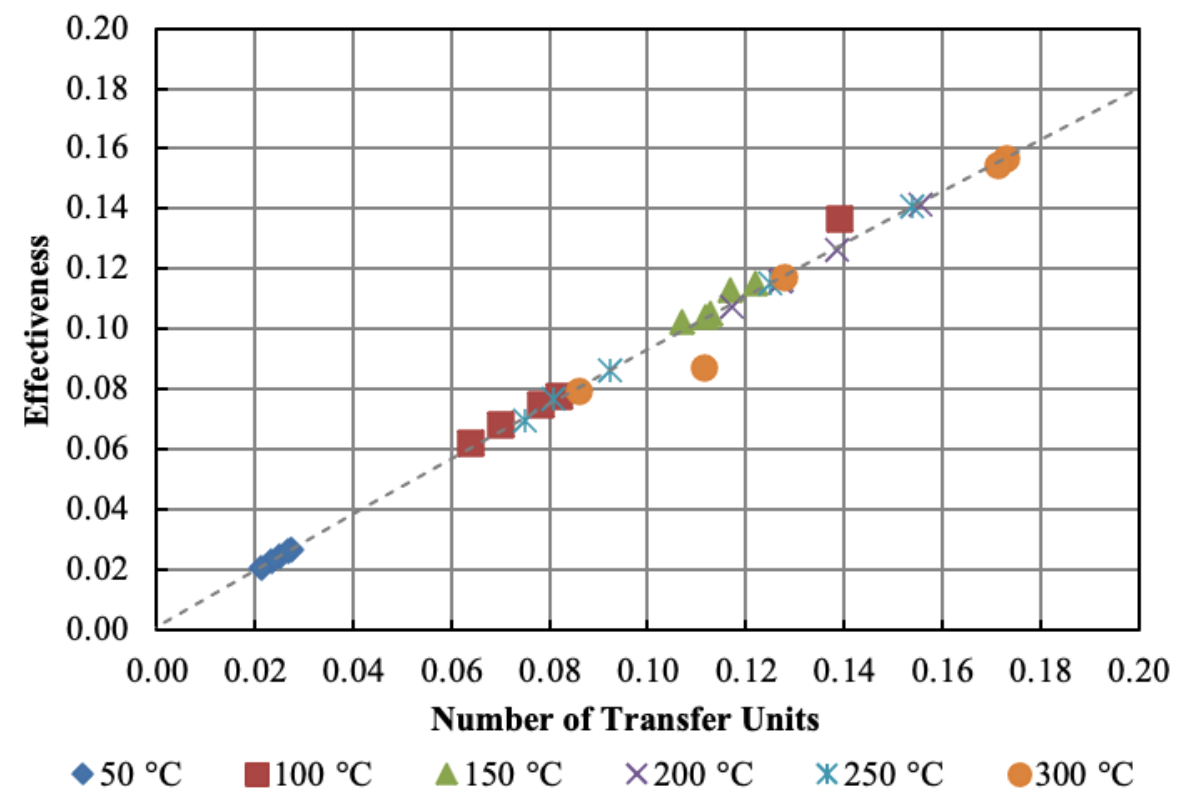

Figure 17: Comparison between the effectiveness of the heat exchanger and the Number of transfer units

\subsection{Comparison of results}

Regarding the temperature at the outlets, the CFD results compared well to the experimental results, with a maximum difference of $10 \%$ at the evaporator section and $15 \%$ at the condenser section as can be seen in Figure 18:

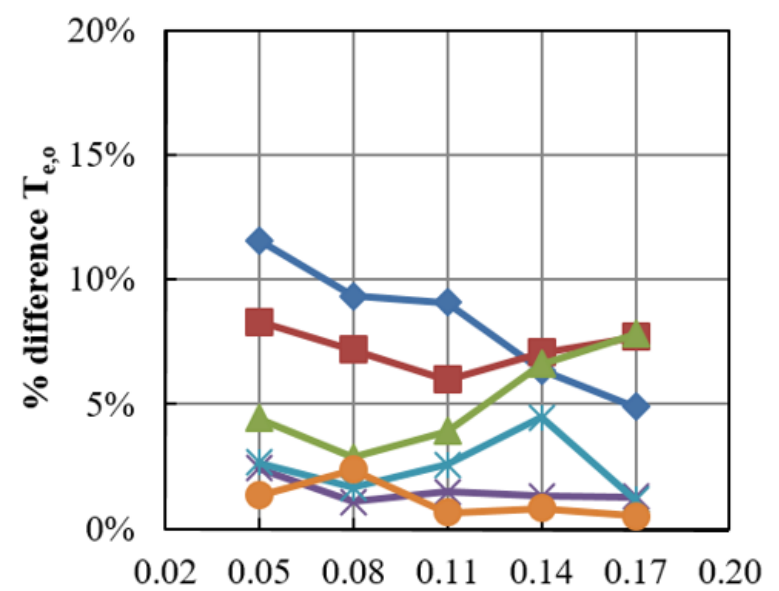

Average inlet air mass flow rate $(\mathrm{kg} / \mathrm{s})$

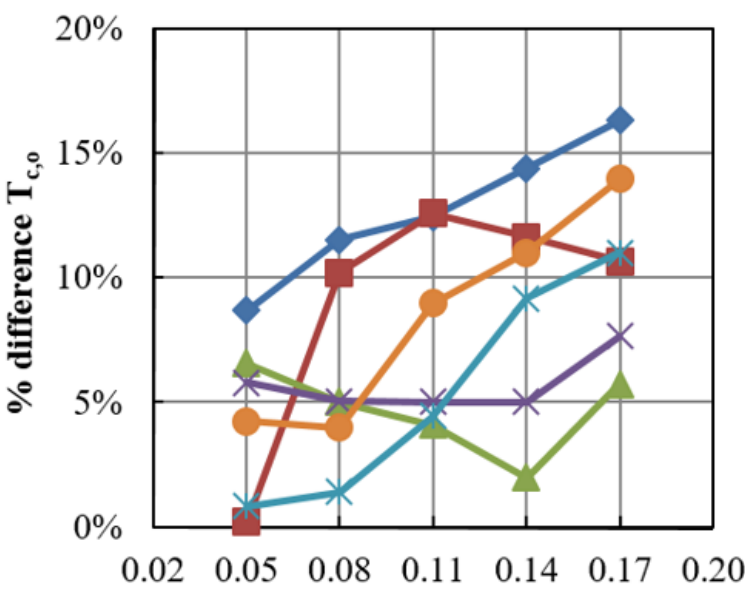

Average inlet air mass flow rate $(\mathrm{kg} / \mathrm{s})$

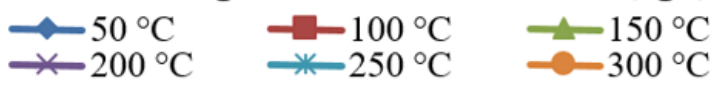

Figure 18: Percentage temperature difference at the outlets between the experimental test and the CFD simulation 
Figure 19 portrays a more detailed comparison between the outlet temperature of the evaporator section and the condenser section; a good agreement is found on both as they tend towards the centre of the graph. According to Figure 19, it is found that the CFD simulation is slightly over-estimating the temperature at the outlet at low evaporator mass flow rates and under-estimating them as the mass flow rates increase.

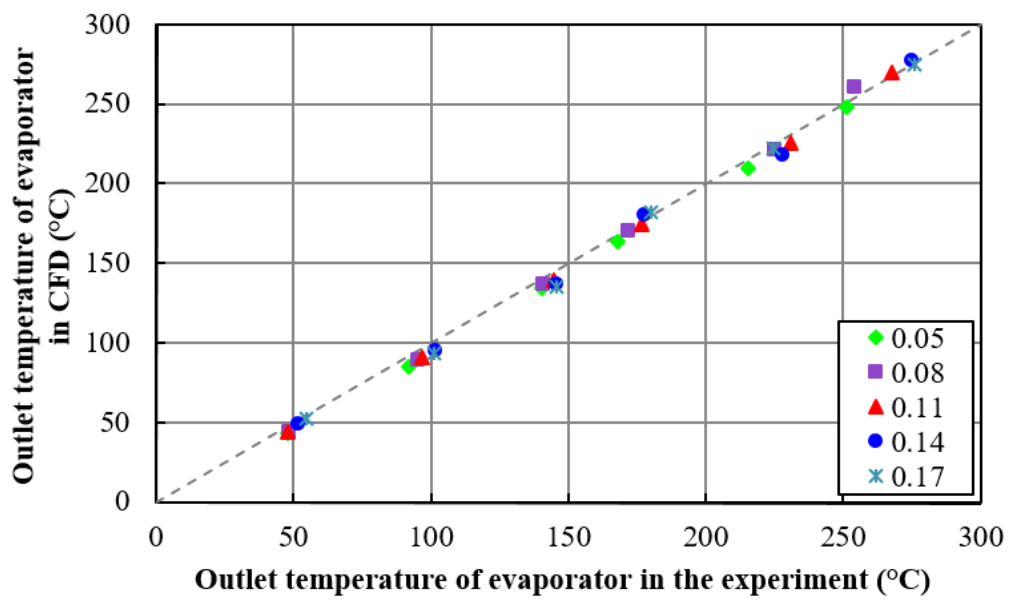

Figure 19: Comparison between experimental and CFD results for the temperature at the outlet of the evaporator at different mass flow rates.

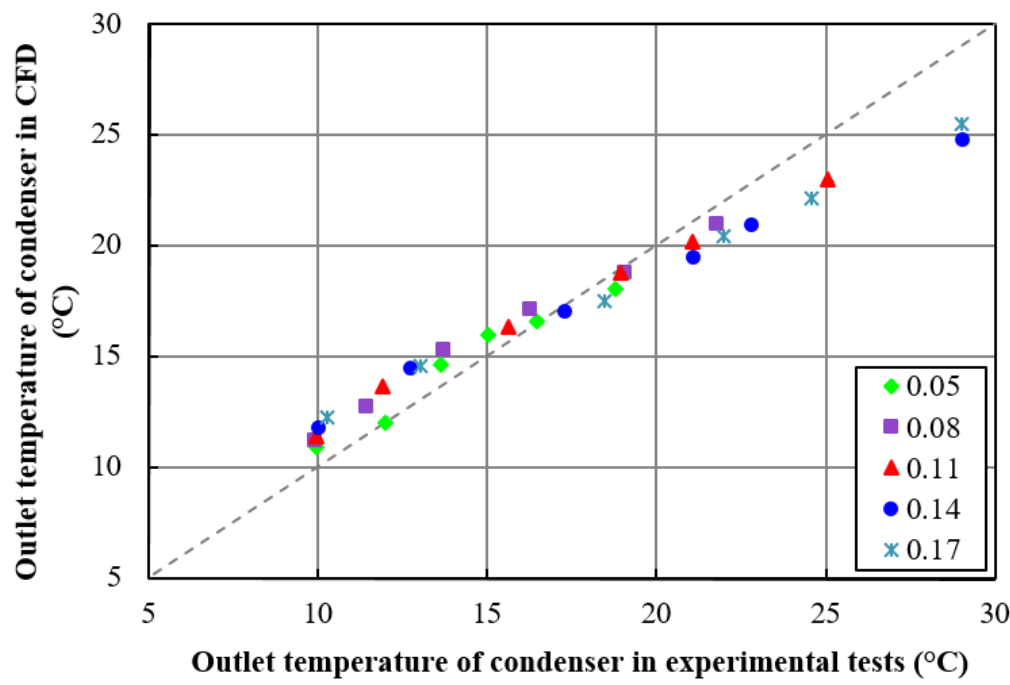

Figure 20: Comparison between experimental and CFD results for the temperature at the outlet of the condenser at different mass flow rates.

The heat transfer rate achieved in the CFD was compared with that found from the experimental results and plotted in Error! Reference source not found.19. Following the same behaviour as Error! Reference source not found.8, the CFD simulation over-estimates the performance of the heat exchanger. However, this is to be expected as in reality it is impossible to have an insulation that is completely adiabatic; particularly with the geometry of the heat exchanger under study. The CFD simulation also appears to be slightly under-estimating the performance of the heat exchanger at higher mass flow rates (10\%); this may be due to higher inlet turbulence in the experimental rig as a result of an increased mass flow rate which is impossible to predict as an inlet boundary condition for the CFD model. 


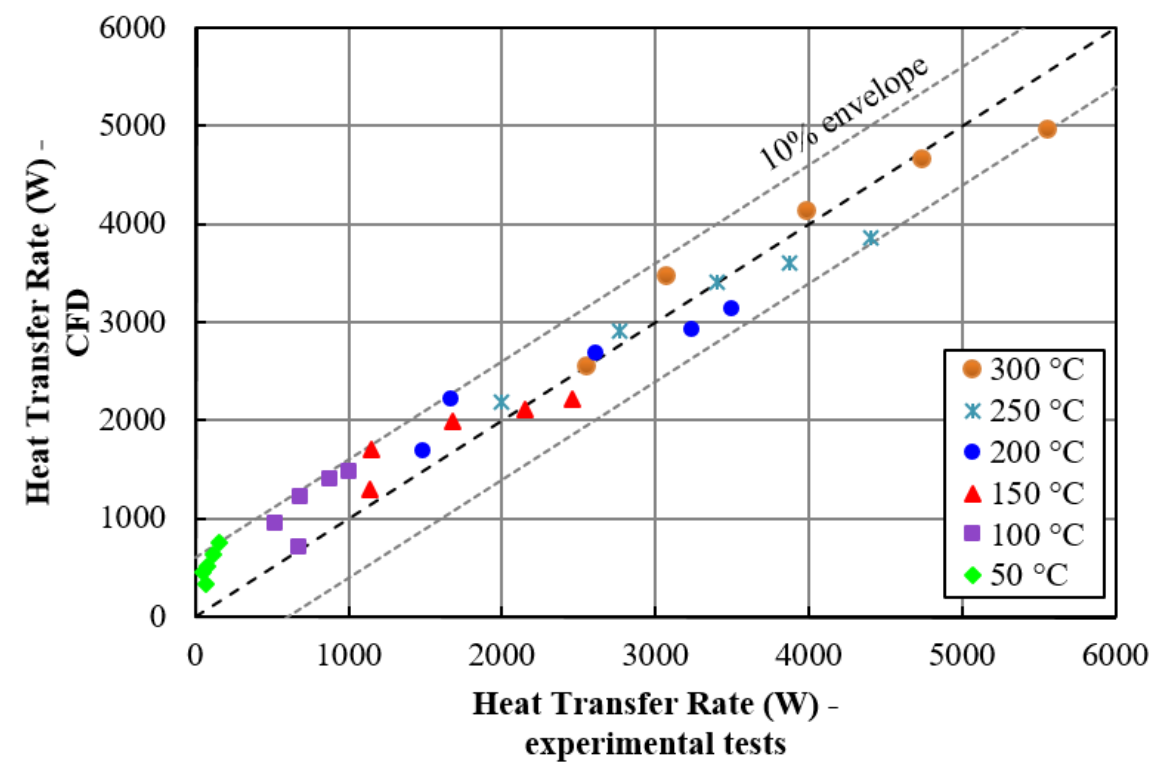

Figure 21: Comparison of the heat transfer rate between the experimental test and the CFD model for different operating temperatures.

The difference in temperature is partially reflected in the heat transfer rate. Error! Reference source not found.19 includes a comparison of the overall heat transfer rate between the experimental tests and CFD simulation. All the results are included within a 10\% fluctuation showing a good correlation between the experimental data and the data obtained from the simulations. The theoretical results were plotted in a similar-type graph in Figure 22 and it was observed that the CFD simulation clearly follows the same trend. This leads to the conclusion that the inherent error is caused by the theoretical expressions which may require some fine tuning in order to better adapt to the problem at hand.

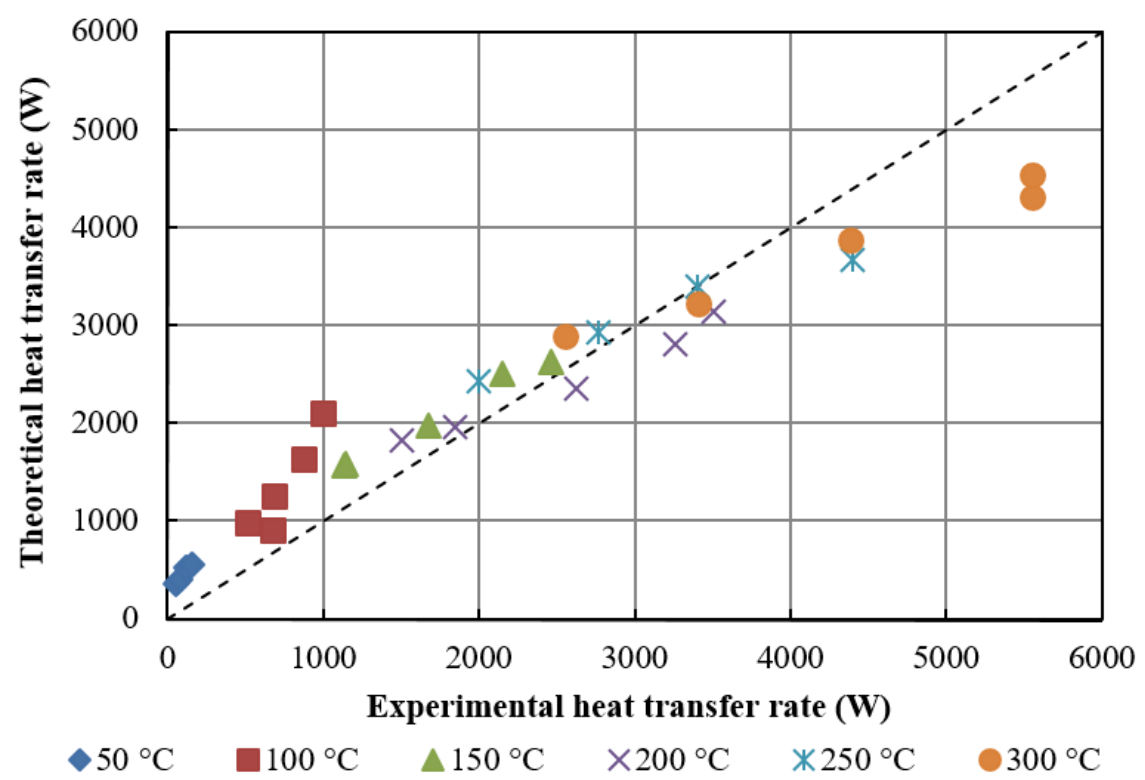

Figure 22: Direct comparison between the experimental and the predicted heat transfer rate.

\subsection{Results from CFD simulation}

CFD was useful as the visualisation of the problem allowed the identification of recirculation zones within the unique geometry of the heat exchanger. Figure 23 illustrates a vector plot of the velocity within the evaporator section of the heat exchanger. The higher velocities were found in the vicinity 
of the heat pipes. In this vector plot, the recirculation zones are clearly identifiable in blue at the top and bottom of the figure.

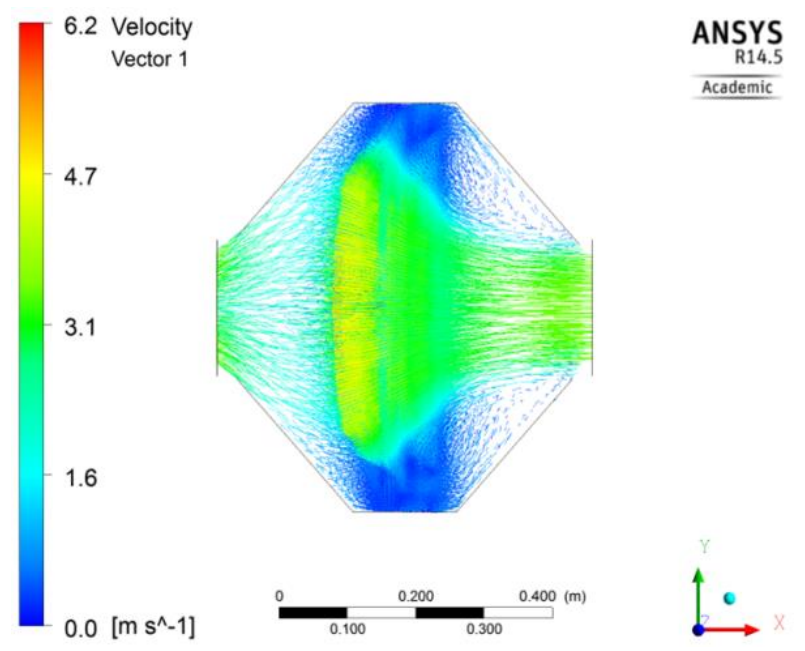

Figure 23: Vector velocity plot of the evaporator section

Figure 24 presents the average velocity profile within the condenser section. The inlet temperature and mass flow rate were kept constant in the condenser section allowing Figure 24 to represent the condenser section for all of the conditions tested. The blue areas represent re-circulation, common after the cylinders in parallel arrangements.

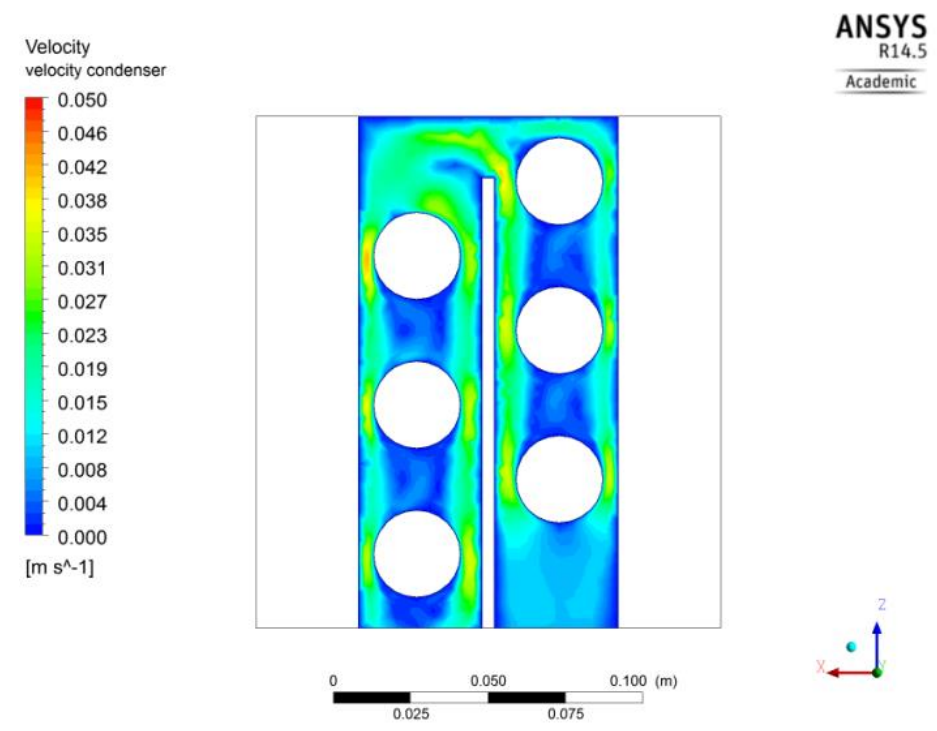

Figure 24: Velocity profile within the condenser section of the heat exchanger

A temperature contour of the evaporator is shown in Figure 25; the air is entering the evaporator at $300^{\circ} \mathrm{C}$ from the left and leaves the evaporator at approximately $255^{\circ} \mathrm{C}$. Temperatures lower than $250^{\circ} \mathrm{C}$ are shown within the pipes. The variation of the temperature on the hot flow is particularly noticeable in this figure. The temperature of the thermosyphons at that particular height is also shown in its respective location. 


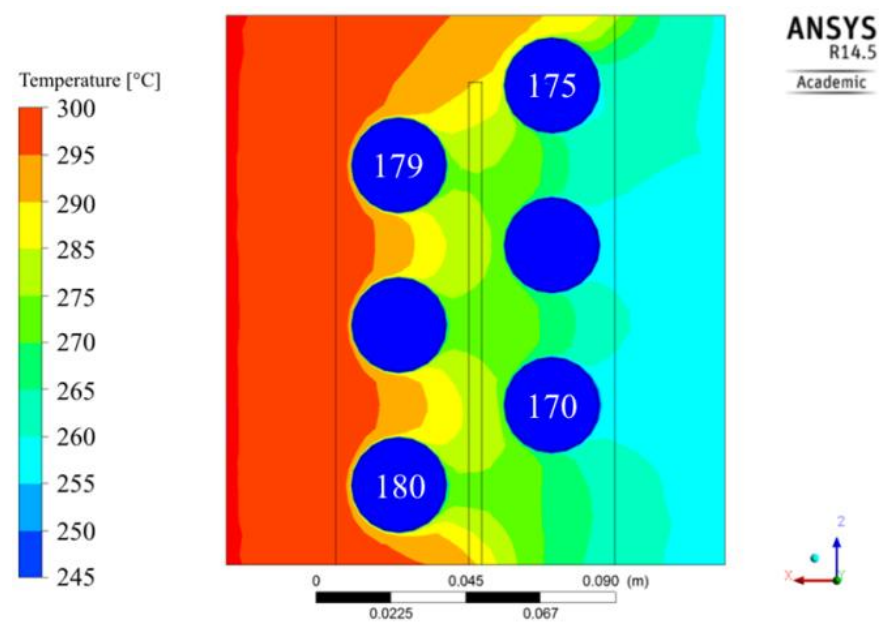

Figure 25: Average temperature contour of the air in the evaporator section at $300^{\circ} \mathrm{C}$ and $0.08 \mathrm{~m} / \mathrm{s}$.

Figure 26 illustrates the temperature contour of the condenser section for the same inlet conditions as Figure 25 . The water enters the condenser at $10^{\circ} \mathrm{C}$ and leaves the condenser at approximately $20^{\circ} \mathrm{C}$. The numbers in the pipes represent the temperatures of the pipes at the condenser section. It can be observed that they diverge from the temperatures shown in Figure 25 and that is because the pipes are not isothermal within as a normal thermosyphon would be; their temperature varies along the $y$-axis due to the different fluid temperatures surrounding the pipes. The overall average temperature of the pipes, however, coincided with the saturation temperature of the pipes.

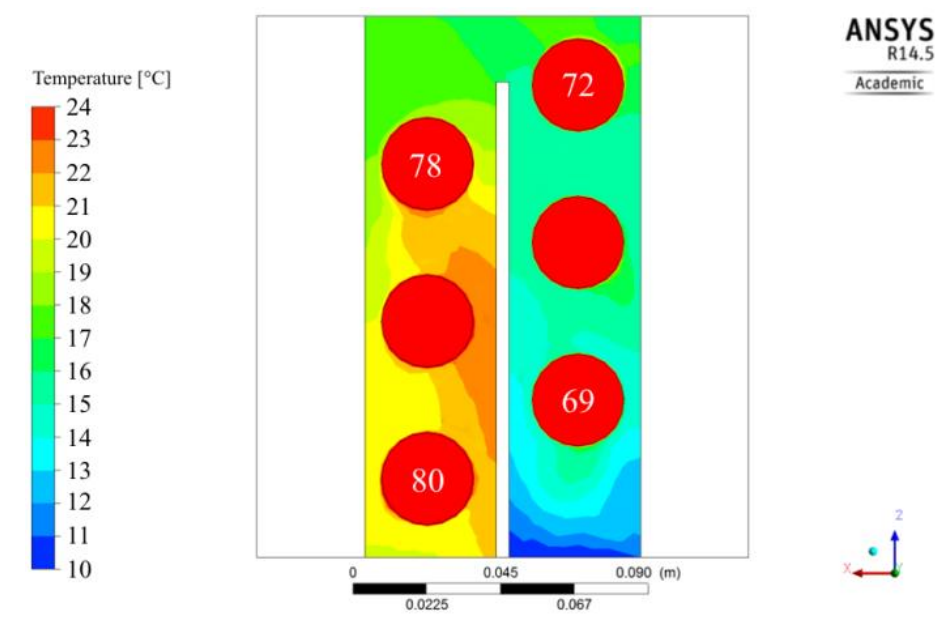

Figure 26: Average temperature contour of the water in the condenser for the evaporator conditions of $300^{\circ} \mathrm{C}$ and $0.08 \mathrm{~m} / \mathrm{s}$

\subsection{Error Propagation}

An uncertainty study was conducted on the error propagation from the measurement instruments used in the experimental rig and the results are presented in Figure 27. The method is explained thoroughly in section 4.3.4. 


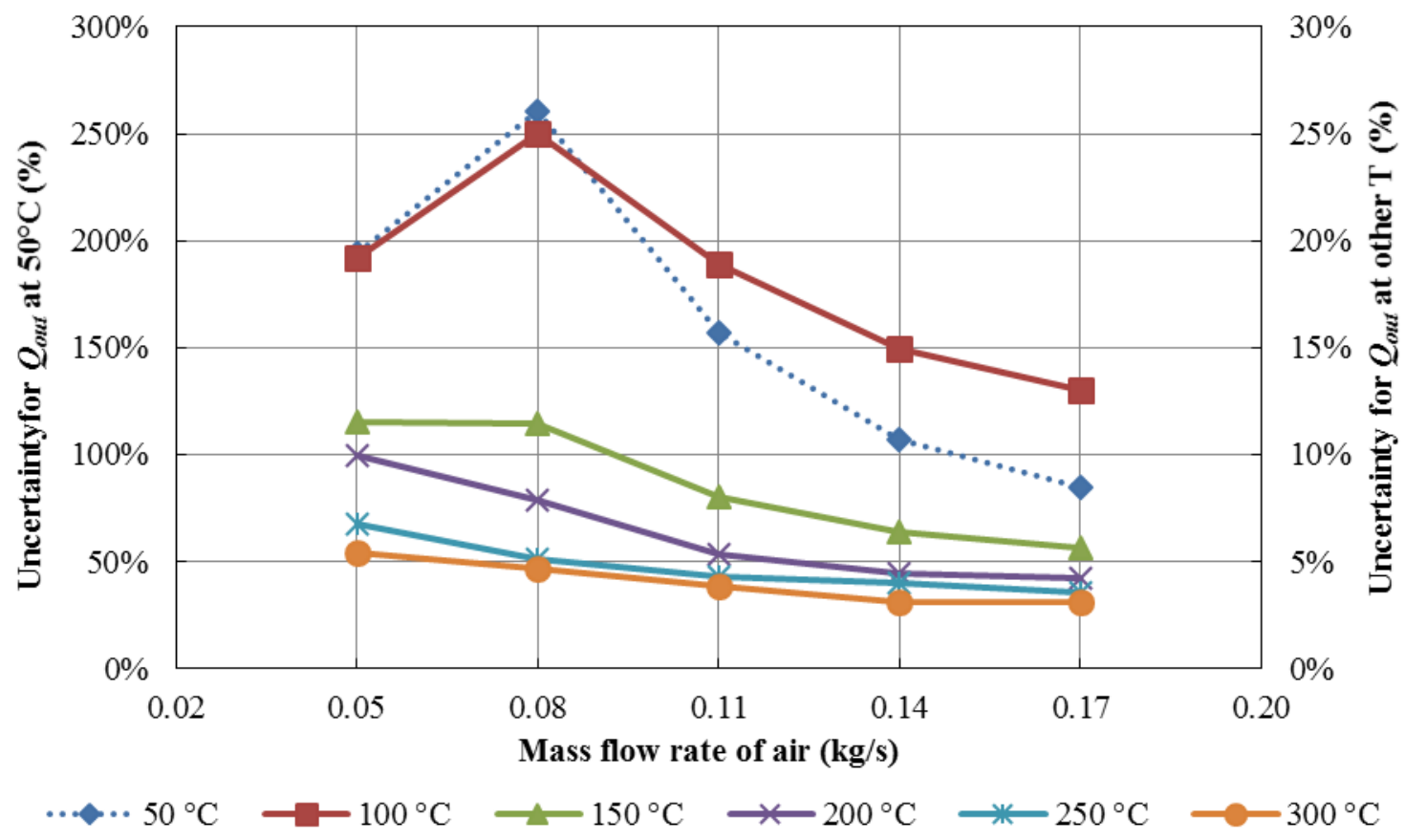

Figure 27: Error propagation for $Q_{\text {out }}$

It was observed that the smaller the difference in temperature between the inlet and the outlet temperatures, the higher the uncertainty. This is particularly striking at $50^{\circ} \mathrm{C}$ inlet air temperature where the uncertainty hovers the $300 \%$ due to the fact that the temperature variation is less than $1{ }^{\circ} \mathrm{C}$. At $100{ }^{\circ} \mathrm{C}$ the $\Delta T_{c}$ already fluctuates close to $2^{\circ} \mathrm{C}$ and therefore the uncertainty propagation is reduced. For all the other tests the uncertainty when determining the $Q_{\text {out }}$ is lower than $10 \%$ and stays within the 5\% range, which is a more acceptable range for most engineering applications.

Overall the trend is for the error propagation to reduce as the mass flow rate and the difference in temperatures increase, a trend seen in all the tests.

\section{Conclusions}

An experimental and a numerical investigation of a heat pipe-based heat exchanger was successfully carried out and verified through comparison with a real-world test experiment. The following conclusions were obtained according to the results:

- Higher temperatures and mass flow rates result in higher heat transfer rates up to a limit of 900W/pipe.

- Equations found in the literature seem to over-predict the results at low evaporator temperatures and under-predict at higher evaporator temperatures - an update is suggested.

- The modelling of the thermosyphons as solid bodies with a conductivity extracted from an analytical study involving the $\varepsilon$-NTU method has been tried and tested in a CFD simulation and the results proved very satisfactory.

- Good agreement between the experimental and numerical results for all temperatures was found for a wide range of flow conditions on the evaporator side; an average 5\% temperature difference was observed between the numerical and experimental results in the evaporator section and $7 \%$ in the condenser section.

\section{Acknowledgements}

The reported work was funded by the European Development Fund and Econotherm (UK) Limited. 


\section{References}

[1] C. Haddad, C. Périlhon, A. Danlos, M.-X. François and G. Descombes, "Some Efficient Solutions to Recover Low and Medium Waste Heat: Competitiveness of the Thermoacoustic Technology," Energy Procedia, vol. 50, pp. 1056-1069, 2014.

[2] Y. H. Yau, "The use of a double heat pipe heat exchanger system for reducing energy consumption of treating ventilation air in an operating theatre-A full year energy consumption model simulation," Energy and Buildings, vol. 40, no. 5, pp. 917-925, 2008.

[3] T. D. Swanson and G. C. Birur, "NASA thermal control technologies for robotic spacecraft," Applied Thermal Engineering, vol. 23, no. 9, pp. 1055-1065, June 2003.

[4] J. Choi, M. Jeong, J. Yoo and M. Seo, "A new CPU cooler design based on an active cooling heatsink combined with heat pipes," Applied Thermal Engineering, vol. 44, pp. 50-56, November 2012.

[5] H. Jouhara and R. Meskimmon, "Heat pipe based thermal management systems for energyefficient data centres," Energy, vol. 77, pp. 265-270, 2014.

[6] A. Faghri, "Heat pipes: Review, Opportunities and Challenges," Frontiers in Heat Pipes, vol. 5, no. 1, pp. 1-48, 2014.

[7] D. Reay and P. Kew, Heat pipes: Theory, Design and Applications, Elsevier Limited, 2006.

[8] C. Han and L. Zou, "Study on the heat transfer characteristics of a moderate-temperature heat pipe heat exchanger," International Journal of Heat and Mass Transfer, vol. 91, pp. 302-310, 2015.

[9] H. Shabgard, M. J. Allen, N. Sharifi, S. P. Benn, A. Faghri and T. L. Bergman, "Heat pipe heat exchangers and heat sinks: Opportunities, challenges, applications, analysis, and state of the art," International Journal of Heat and Mass Transfer, vol. 89, pp. 138-158, 2015.

[10] H. Jouhara and R. Meskimmon, "Experimental investigatuion of wraparound loop heat pipe heat exchanger used in energy efficient air handling units," Energy, vol. 35, no. 12, pp. 4592-4599, 2010.

[11] A. Faghri, "Review and Advances in Heat Pipe Science and Technology," Journal of Heat Transfer, vol. 134, no. 12, 12 October 2012.

[12] Econotherm, "Econotherm - Waste Heat Recovery \& Recycling Technology," 2012. [Online]. Available: http://www.econotherm.eu/index.html. [Accessed 1 2012].

[13] H. Hagens, F. Ganzevles, C. van der Geld and M. Grooten, "Air heat exchangers with long heat pipes: Experiments and predictions," Applied Thermal Engineering, no. 27, pp. 2646-2434, 2007.

[14] S. Noie, "Heat transfer characteristics of a two-phase closed thermosyphon," Applied Thermal Engineering 25, pp. 495-506, 2005.

[15] A. Nuntaphan, J. Tiansuwan and T. Kiatsiriroat, "Heat Transfer Coefficients of Thermosyphon Heat Pipe at Medium Operating Temperature," Thailand, 2001.

[16] L. Vasiliev, "Heat Pipes in modern heat exchangers," Elsevier, Belarus Minsk, Russia, 2005.

[17] J. T. Cieslinski and A. Fiuk, "Heat transfer characteristics of a two-phase thermosyphon heat exchanger," Applied Thermal Engineering, vol. 51, no. 1-2, pp. 112-118, 2012.

[18] Y. Yau and M. Ahmadzadehtalatapeh, "A review on the application of horizontal heat pipe heat exchangers in air conditioning systems in the tropics," Applied Thermal Engineering, vol. 30, no. 2-3, pp. 77-84, 2010.

[19] M. Ahmadzadehtalatapeh, "An air-conditioning system performance enhancement by using heat pipe based heat recovery technology," Scientia Iranica, vol. 20, no. 2, pp. 329-336, April 2013.

[20] T. Jadhav and M. Lele, "Theoretical energy saving analysis of air conditioning system using heat pipe heat exchanger for Indian climatic zones," Engineering Science and Technology, an International Journal, vol. 18, no. 4, pp. 669-673, 2015.

[21] F. Yang, X. Yuan and G. Lin, "Waste heat recovery using heat pipe heat exchanger for heating automobile using exhaust gas," Applied Thermal Engineering, vol. 23, no. 3, pp. 367-372, 2003. 
[22] Y. Wang, X. Han, Q. Liang, W. He and Z. Lang, "Experimental investigation of the thermal performance of a novel concentric condenser heat pipe array," International Journal of Heat and Mass Transfer, vol. 82, pp. 170-178, March 2015.

[23] W. Srimuang and P. Amatachaya, "A review of the applications of heat pipe heat exchangers for heat recovery," Renewable and Sustainable Energy Reviews, vol. Volume 16, no. Issue 6, pp. 4303-4315, 2012.

[24] S. Noie, "Investigation of thermal performance of an air-to-air thermosyphon heat exchanger using E-NTU method," Applied Thermal Engineering, vol. 26, no. 5-6, pp. 559-567, 2006.

[25] J. Danielewicz, M. A. Sayegh, B. Sniechowska, M. Szulgowska-Zgrzywa and H. Jouhara, "Experimental and analytical performance investigation of air to air two phase closed themrosyphon based heat exchangers," Energy, vol. 77, pp. 82-87, December 2014.

[26] H. Jouhara and H. Merchant, "Experimental investigation of a thermosyphon based heat exchanger used in energy efficient air handling units," Energy, pp. 82-89, 2012.

[27] A. Alizadehdakhel, M. Rahimi and A. Alsairafi, "CFD modeling of flow and heat transfer in a thermosyphon," International Communicaitons in Heat and Mass Transfer 37, pp. 312-318, 2010.

[28] B. Fadhl, L. C. Wrobel and H. Jouhara, "Numerical modelling of the temperature distribution in a two-phase closed thermosyphon," Applied Thermal Engineering, vol. 60, no. 1-2, pp. 122-131, 2013.

[29] H. Jouhara, B. Fadhl and L. C. Wrobel, "Three-dimensional CFD simulation of geyser boiling in a two-phase closed thermosyphon," International Journal of Hydrogen Energy, p. In press, 2016.

[30] K. Negishi and T. Sawada, "Heat transfer performance of an inclined two-phase closed thermosyphon," International Journal of Heat and Mass Transfer, vol. 26, pp. 1207-1213, 1983.

[31] I. Khazaee, R. Hosseini and S. Noie, "Experimental investigation of effective parameters and correlation of geyser boiling in a two-phase closed thermosyphon," Applied Thermal Engineering, vol. 30, no. 5, pp. 406-412, 2010.

[32] H. Shabgard, B. Xiao, A. Faghri, R. Gupta and W. Weissman, "Thermal characteristics of a closed thermosyphon under various filling conditions," International Journal of Heat and Mass Transfer, vol. 70, pp. 91-102, 2014.

[33] B. Hughes, H. Chaudhry and J. Calautit, "Passive energy recovery from natural ventilation air streams," Applied Energy, vol. 113, pp. 127-140, 2014.

[34] B. Selma, M. Désilets and P. Proulx, "Optimization of an industrial heat exchanger using an open-source CFD code," Applied Thermal Engineering, vol. 69, no. 1-2, pp. 241-250, 2014.

[35] H. Peng, X. Ling and J. Li, "Numerical simulation and experimental verification on thermal performance of a novel fin-plate thermosyphon," Applied Thermal Engineering, vol. 40, pp. 181188, 2012.

[36] J. K. Calautita, H. N. Chaudhrya, B. R. Hughesa and S. A. Ghanib, "Comparison between evaporative cooling and a heat pipe assisted thermal loop for a commercial wind tower in hot and dry climatic conditions," Applied Energy, vol. 101, pp. 740-755, 2013.

[37] H. Mroué, J. Ramos, L. Wrobel and H. Jouhara, "Experimental and numerical investigation of an air-to-water heat pipe-based heat exchanger," Applied Thermal Engineering, vol. 78, no. ISSN 1359-4311, pp. 339-350, 2015.

[38] E. Azad and F. Geoola, "A design procedure for gravity assisted heat pipe heat exchanger," Heat Recovery System, vol. 4, no. 2, pp. 101-111, 1984.

[39] W. Kays and A. London, Compact Heat Exchanger Design, third ed. ed., New York: McGrawHill, 1984.

[40] A. Lukitobudi, A. Akbarzadeh, P. Johnson and P. Hendy, "Design, construction and testing of a thermosyphon heat exchanger for medium temperature heat recovery in bakeries," Heat Recovery Systems and CHP, vol. 15, no. 5, pp. 481-491, 1995.

[41] A. Zhukauskas, "Heat Transfer from Tubes in Cross Flow," in Advances in Heat Transfer, J. H. 
a. T. Irvine, Ed., New York, Academic Press, 1972.

[42] Y. A. Çengel, in Heat Transfer: A Practical Approach, McGraw-Hill, 2002, pp. 7, 515.

[43] A. Faghri, Heat Pipe Science and Technology, Taylor \& Francis, 1995.

[44] W. Rohsenow, J. Hartnett and Y. Cho, Handbook of Heat Transfer, third ed. ed., McGraw-Hill, 1998.

[45] J. Ramos, A. Chong and H. Jouhara, "Numerical investigation of a cross flow air-to-water heat pipe-based heat exchanger used in waste heat recovery," SusTEM 2015, Newcastle, 2015.

[46] W. M. Rohsenow, "A method of correlating heat-transfer data for surface boiling of liquids," Trans. ASME, vol. 74, pp. 969-976, 1952.

[47] F. Incropera and D. DeWitt, Fundamentals of Heat and Mass Transfer, Fourth Edition ed., New York: John Wiley \& Sons, 1996.

[48] W. McAdams, Heat Transmission, New York: McGraw Hill, 1954.

[49] ANSYS, “Ansys Fluent 14.5 Help,” 2012-2014. [Online]. [Accessed 2015].

[50] K. Ekambara, R. Sanders, K. Nandakumar and J. Masliyah, "CFD simulation of bubbly twophase flow in horizontal pipes," Chemical Engineering Journal, vol. 144, no. 2, pp. 277-288, 2008 .

[51] Fluent Inc., "FLUENT 6.3 User's Guide," 2014.

[52] J. Ramos, A. Chong, C. K. Tan, J. Matthews, M. A. Boocock and H. Jouhara, "Experimental Analysis of gas to water two phase closed thermosyphon based heat exchanger," 10th International Conference on Heat Transfer, Fluid Mechanics and Thermodynamics, Orlando, USA, 2014. 